$\begin{array}{ll}\text { Research Square } & \begin{array}{l}\text { Preprints are preliminary reports that have not undergone peer review. } \\ \text { They should not be considered conclusive, used to inform clinical practice, } \\ \text { or referenced by the media as validated information. }\end{array}\end{array}$

\title{
Optimized Dose Selective HDAC Inhibitor Tucidinostat Overcomes Anti-PD-L1 Antibody Resistance in Experimental Solid Tumors
}

\section{Pei Zhang}

National Cancer Center, Chinese Academy of Medical Sciences \& Peking Union Medical College Yang Du

Chinese Academy of Sciences

\section{Hua Bai}

National Cancer Center, Chinese Academy of Medical Sciences \& Peking Union Medical College

\section{Zhijie Wang}

National Cancer Center, Chinese Academy of Medical Sciences \& Peking Union Medical College

Jianchun Duan

National Cancer Center, Chinese Academy of Medical Sciences \& Peking Union Medical College

\section{Xin Wang}

National Cancer Center, Chinese Academy of Medical Sciences \& Peking Union Medical College

Jia Zhong

National Cancer Center, Chinese Academy of Medical Sciences \& Peking Union Medical College

\section{Rui Wan}

National Cancer Center, Chinese Academy of Medical Sciences \& Peking Union Medical College Jiachen Xu

National Cancer Center, Chinese Academy of Medical Sciences \& Peking Union Medical College Xiran He

Capital Medical University, Thoracic Tumor Research Institute

\section{Di Wang}

National Cancer Center, Chinese Academy of Medical Sciences \& Peking Union Medical College Kailun Fei

National Cancer Center, Chinese Academy of Medical Sciences \& Peking Union Medical College

\section{Ruofei Yu}

National Cancer Center, Chinese Academy of Medical Sciences \& Peking Union Medical College

Jie Tian

Chinese Academy of Sciences

Jie Wang ( $\square$ zlhuxi@163.com)

National Cancer Center, Chinese Academy of Medical Sciences \& Peking Union Medical College 


\section{Research Article}

Keywords: Tucidinostat, Tumor microenvironment, PD-L1, CCL5, Solid tumor.

Posted Date: February 8th, 2022

DOI: https://doi.org/10.21203/rs.3.rs-1331646/v1

License: (c) (i) This work is licensed under a Creative Commons Attribution 4.0 International License. Read Full License 


\section{Abstract}

Background: Although immune checkpoint inhibitors (ICls) have influenced the treatment paradigm for multiple solid tumors, increasing evidence suggests that primary and adaptive resistance may limit the long-term efficacy of ICls. New therapeutic strategies with other drug combinations are hence warranted to enhance the antitumor efficacy of ICls. As a novel tumor suppressor, histone deacetylase inhibitor tucidinostat has been successfully confirmed to act against hematological malignancies. However, the underlying mechanisms of action for tucidinostat and whether it can manipulate the tumor microenvironment (TME) in solid tumors remain unclear.

Methods: Three murine tumor models were developed to define the significant role of different doses of tucidinostat in TME. The immunotherapeutic effect of tucidinostat combined with anti-programmed cell death ligand 1 antibody (aPD-L1) was demonstrated. Furthermore, the effect of tucidinostat on phenotypic characteristics of peripheral blood mononuclear cells from lung cancer patients was investigated.

Results: With an optimized dose, tucidinostat could alter TME and promote the migration and infiltration of $\mathrm{CD}^{+} \mathrm{T}$ cells into tumors, partially by increasing the activity of $\mathrm{C}-\mathrm{C}$ motif chemokine ligand 5 via NF-KB signaling. Moreover, tucidinostat significantly promoted M1 polarization of macrophages and increased the in vivo antitumor efficacy of aPD-L1. Tucidinostat also enhanced the expression of the costimulatory molecules on human monocytes, suggesting a novel and improved antigen-presenting function.

Conclusions: A combination regimen of tucidinostat and APD-L1 may work synergistically to reduce tumor burden in patients with cancer by enhancing the immune function and provided a promising treatment strategy to overcome $\mathrm{ICl}$ treatment resistance.

\section{Background}

Programmed cell death protein 1 (PD-1) is an immune checkpoint receptor expressed on activated T cells that modulate tissue immune tolerance[1, 2]. Tumor cells frequently overexpress the ligand programmed death ligand-1 (PD-L1), thereby facilitating their escape from immune surveillance[3-5]. Monoclonal antibodie (mAb)s against PD-1 or PD-L1 have demonstrated remarkable clinical efficacy in patients with a variety of cancers[3]. However, accumulating evidence suggests that mAbs against PD-1 and PD-L1 are less effective in non-inflamed tumors, indicating that such tumors are resistant to immune attack. Indeed, tumors unresponsive to PD-1 or PD-L1 mAbs are characterized by poor lymphocyte infiltration, low PD-L1 expression, and increased immunosuppressive factor expression in the tumor microenvironment (TME). Combining PD-1 or PD-L1 mAbs with certain agents that can modulate the immunosuppressive state may overcome the primary and adaptive resistance[6, 7]. Cytotoxic chemotherapy or molecularly targeted therapy has been demonstrated to enhance the effect of PD-1/PD-L1 mAbs as immunotherapeutic drugs[8-10]. 
Several studies have suggested a bidirectional relationship between epigenetic modifications and antitumor immunity in TME[11-13]. Cancers can be caused not only due to a change in the genomic DNA sequence but also through two typical epigenetic modifications: DNA methylation and histone modification[14]. These epigenetic modifications remodel the chromatin structure, thereby altering the gene expression profile and cell phenotype and potentially resulting in cell cycle dysregulation and tumor development[15-17]. Conversely, the reversal of histone and non-histone protein acetylation by histone deacetylase (HDAC) inhibitors can induce cell cycle arrest, differentiation, and cancer cell death[18, 19]. Such antitumor effects of HDAC inhibitors have been proven in human hematological tumors, but not yet in solid tumors. Recently, preclinical studies have reported the efficacy of HDAC inhibitors combined with other therapeutic agents, including chemotherapy or targeted drugs, in treating solid tumors[20-22]. Trials investigating these combinations in patients with solid tumors are also ongoing, and the preliminary data obtained seem promising. However, the antitumor effects of HDAC inhibitors combined with immune checkpoint inhibitors (ICls) have not been extensively studied[23, 24]. Challenges remain in harnessing the full potential of combined HDAC inhibition and immunotherapy and selecting optimal regimens for different solid tumors.

Tucidinostat, an HDAC inhibitor belonging to the oral benzamide class and having specificity for HDAC1, HDAC2, HDAC3, and HDAC10 subtypes, has been approved for the treatment of relapsed or refractory peripheral T cell lymphoma and is under clinical development globally for various other neoplastic and non-neoplastic diseases[25-28]. A recent phase III trial reported that tucidinostat combined with endocrine blockade could be effective against advanced hormone receptor-positive HER2-negative breast cancer progression after endocrine therapy alone[29]. Additionally, grade 3 or 4 toxicities including neutropenia, leucopenia, and thrombocytopenia were more frequent in the tucidinostat group than in the placebo group, indicating that tucidinostat may induce immunosuppression, which is not conducive to effective immunotherapy. As we know, low-dose chemotherapeutic drugs have been shown to remodel the tumor immune microenvironment and provide benefits when combined with ICIs[30-33]. Therefore, assessing the effect of different doses of tucidinostat in the presence or absence of ICls on solid tumor growth and TME immune status is warranted to discover an optimized combination therapy.

Here, we analyzed the antitumor efficacy of different doses of tucidinostat alone and in combination with aPD-L1 in three murine solid tumor models that were unresponsive or transiently responsive to ICls to explore the optimal strategy for combining tucidinostat and aPD-L1, as well as its underlying mechanisms. This may provide guidance to improve the clinical management of combined immunotherapy.

\section{Materials And Methods}

\section{Materials and Reagents}

Tucidinostat (Cat\# HY-109015), vorinostat (Cat\# HY-10221), TMP-195 (Cat\# HY-18361), and NF-KB inhibitor BAY11-7082 (Cat\# HY-13453) were obtained from MCE (Monmouth Junction, NJ, USA). 
Dimethyl sulfoxide (DMSO) have been used as vehicle control for each drug.

\section{Cell culture}

4T1 breast cancer cells, Lewis lung cancer (LLC) cells, CT26 colorectal cancer cells, and Raw 264.7 cells were purchased from Chinese Academy of Sciences (Beijing, China) and cultured in RPMI-1640 medium (Hyclone) containing $10 \%$ fetal bovine serum at $37^{\circ} \mathrm{C}$ in a $5 \% \mathrm{CO}_{2}$ incubator.

\section{Establishment of murine syngeneic tumor models}

For subcutaneous injections, $5 \times 10^{5}$ mouse tumor cells were injected into the right flank of each mouse. When established tumors were palpable 7 days after tumor cell inoculation, mice were treated with different doses of tucidinostat (MCE, gavage, $12.5,25,75 \mathrm{mg} / \mathrm{kg}$, oral gavage, daily) and aPD-L1 (BioXcell, Cat\# BE0101; $200 \mu \mathrm{g}$, intraperitoneal injection, every 3 days). Depletion of CD8 ${ }^{+} \mathrm{T}$ cells was performed by intraperitoneal injection of anti-mouse CD8a (BioXcell, Cat\# BP0117; $200 \mu \mathrm{g}$, every 3 days). Depletion of macrophage was performed by intraperitoneal injection of Clodronate liposomes (FormuMax, Cat\# F70101C-A; 1.4 mg/20g body weight, every 3 days), respectively. The volume of tumor nodules was measured every 3 days and calculated as $V=\left(a \times b^{2}\right) / 2$, where " $a$ " and " $b$ " are the long and short axis of the tumor nodule, respectively. Tumors volume were measured with calipers every three days. Mice were monitored until their individual tumor volume reaches the approved protocol volume limit $\left(2000 \mathrm{~mm}^{3}\right)$. At the treatment, the tumor-bearing mice were anesthetized and tissues were harvested for further analysis.

In the previous human clinical trial, $30 \mathrm{mg}$ tucidinostat was given orally twice a week for 4 consecutive weeks in a 4-week cycle. After translating the drug dosage from human to mice[34], the clinical dose of tucidinostat in mice was near $75 \mathrm{mg} / \mathrm{kg}$ daily.

Animal studies were conducted in accordance with the $\mathrm{NIH}$ animal use guidelines and approved by the Institutional Review Board of National Cancer Center/National Clinical Research Center for Cancer/Cancer Hospital, Chinese Academy of Medical Sciences \& Peking Union Medical College (Permit Number, NCC2020A167).

\section{Quantitative real-time PCR (RT-qPCR)}

Total RNAs were extracted using the RNeasy Kit (Takara Bio). The qRT-PCR was carried out using SYBR Green Premix Ex TaqTM II (Takara Bio) on a ABI StepOnePlus Real-Time PCR Detection System (Thermo Fisher Scientific). Results were normalized to the housekeeping gene GAPDH. Relative gene expression level from different groups were calculated with the 2- $\Delta \Delta C T$ method and compared with the expression level of appropriate control cells.

Specific primer sequences for individual genes were as follows: CCL5(forward:5'GTATTTCTACACCAGCAGCAAG-3';reverse:5'-TCTTGAACCCACTTCTTCTCTG-3');CXCL9(forward:5'- 
AATCCCTCAAAGACCTCAAACA-3';reverse:5'-TCCCATTCTTTCATCAGCTTCT-3');CXCL10(forward:5'CAACTGCATCCATATCGATGAC-3';reverse:5'-GATTCCGGATTCAGACATCTCT-3');PD-L1(forward:5'TGAGCAAGTGATTCAGTTTGTG-3';reverse:5'-CATTTCCCTTCAAAAGCTGGTC-3');iNOS(forward:5'GCCGAGTGCAAGCATGGAGAG-3';reverse:5'-GGCTGTGAGGTGAGGTTGAAGAAG-3');CD86(forward:5'ACGGAGTCAATGAAGATTTCCT-3';reverse:5'-GATTCGGCTTCTTGTGACATAC-3');CD206(forward:5'CCTATGAAAATTGGGCTTACGG-3';reverse:5'-CTGACAAATCCAGTTGTTGAGG-3');Arg1(forward:5'CATATCTGCCAAAGACATCGTG-3';reverse:5'-GACATCAAAGCTCAGGTGAATC-3');GAPDH(forward:5'GTATTTCTACACCAGCAGCAAG-3';reverse:5'-TCTTGAACCCACTTCTTCTCTG-3').

\section{Western blot analysis}

The protein was separated by $10 \%$ SDS-PAGE gel and transferred onto a polyvinylidene difluoride membrane (Millipore). Subsequently, the membrane was blocked and incubated overnight at $4^{\circ} \mathrm{C}$ with the primary antibody including anti-CCL5 mAb (1: 500, Abcam, Cat\# ab7198) and anti-phospho-NF-KB p65(1:1000, Cell Signaling Technology, Cat\# 3039). The same membrane was probed for GAPDH (1:10000, Abcam, Cat\# ab8245) as internal control. Finally, the blots were exposed to the ImageQuant LAS 500 system (GE Healthcare).

\section{Flow cytometric analysis}

Tumors from subcutaneous tumor model were harvested for single-cell suspensions using tumor dissociation kit (Miltenyi Biotec $\mathrm{GmbH}$ ). The drainage lymph node (dLN)s were harvested through mechanical dissociation. Dissociated cells were filtered through a $4 \mu \mathrm{m}$ strainer and suspended in phosphate-buffered saline (PBS) supplemented with 1\% FBS. The cells were stained with the following Abs according to the manufacturer's instructions: CD45 (Cat\# 103114), CD3 (Cat\# 100204), CD4 (Cat\# 100414), CD8a (Cat\# 100752), CD25 (Cat\# 101923), CD44 (Cat\# 103031), CD62L (Cat\# 161203), F4/80 (Cat\# 123128), CD11b (Cat\# 101245), MHC-II (Cat\# 107606), CD206 (Cat\# 141708), Gr-1 (Cat\# 108423), CD11c (Cat\# 117329), CD86 (Cat\# 105014), PD-1 (Cat\# 135206),or PD-L1 (Cat\# 124312) (2.5ul, all from BioLegend) were diluted in FACS buffer (Biolegend).

Various immune cells were separated using a gating strategy based on the expression of known lineage markers for lymphocytes $\left(\mathrm{CD} 45^{+}\right)$, Total T cells $\left(\mathrm{CD} 45^{+} \mathrm{CD} 3^{+}\right), \mathrm{CD}^{+} \mathrm{T}$ cells $\left(\mathrm{CD} 45^{+} \mathrm{CD} 3^{+} \mathrm{CD} 4^{+}\right), \mathrm{CD}^{+} \mathrm{T}$ cells $\left(\mathrm{CD} 45^{+} \mathrm{CD}^{+} \mathrm{CD} 8^{+}\right)$, Treg cells $\left(\mathrm{CD} 45^{+} \mathrm{CD} 3^{+} \mathrm{CD} 4^{+} \mathrm{CD} 25^{+}\right)$, Central Memory $\mathrm{T}$ cells $\left(\mathrm{CD}^{+} \mathrm{CD} 4^{+} \mathrm{CD} 44^{+} \mathrm{CD} 62 \mathrm{~L}^{+}\right)$, Effective Memory T cells $\left(\mathrm{CD} 3^{+} \mathrm{CD} 4^{+} \mathrm{CD} 44^{+} \mathrm{CD} 62 \mathrm{~L}^{-}\right)$, Macrophages $\left(\mathrm{CD} 45^{+} \mathrm{CD} 11 \mathrm{~b}^{+} \mathrm{F} 4 / 80^{+}\right), \mathrm{M} 1$ macrophages $\left(\mathrm{CD} 45^{+} \mathrm{CD} 11 \mathrm{~b}^{+} \mathrm{F} 4 / 80^{+} / \mathrm{MHC}^{-} \mathrm{II}^{+}\right), \mathrm{DC}$ cells $\left(\mathrm{CD} 45^{+} \mathrm{CD} 11 \mathrm{~b}^{-}\right.$ CD11 $\left.\mathrm{c}^{+}\right), \mathrm{MDSCs}\left(\mathrm{CD} 45^{+} \mathrm{CD} 11 \mathrm{~b}^{+} \mathrm{Gr}-1^{+}\right)$, and NK cells $\left(\mathrm{CD} 45^{+} \mathrm{CD}^{-} \mathrm{CD} 49 \mathrm{~b}^{+}\right)$.

Data was performed on the Flow cytometers (BD Biosciences) and analyzed using the Flow Jo software (Ashland, OR, USA).

Immunohistochemistry (IHC) 
Tumors were collected and fixed in $4 \%$ formalin. Sections of paraffin-embedded tissues $(4 \mu \mathrm{m})$ were deparaffinized. After antigen retrieval, the sections were incubated with the primary against CD3 (Cat\# ab16669), CD4 (Cat\# ab183685) or CD8 (Cat\# ab217344) (1:200, all from Abcam), and then stained with the HRP linked secondary Abs or fluorophore-conjugated secondary Abs. The cell nucleus were counterstained using hematoxylin or DAPI.

\section{Genomic Analysis}

RNA sequencing (RNA-seq) was performed in tumors treated with tucidinostat or controls. Published mRNA signatures for T cells and other cell clusters were analyzed[35, 36]. Hierarchical clustering and Sig Clust were used to identify metagenes of statistical significance and data interpretation.

\section{Mice peripheral blood preparation and Cytokine assay}

Peripheral blood were collected from the inner canthus of the experimental mice. Mice white blood cell (WBC) count, red blood cell (RBC) count, platelet (PLT) count, and lymphocytes count were detected by fully automatic hematology analyzer (BC-2800 Vet, Mindray).

Peripheral blood were collected and then centrifuged at 3,000 rpm for $10 \mathrm{~min}$ to isolate the serum. The serum alanine transaminase (ALT), alanine transaminase (ALT), and urea nitrogen (BUN) (all from Anoric Bio-technology) were measured using ELISA kits. The serum IL-10 (Cat\# 431417), IFN-y (Cat\# 430807), TNF-a (Cat\# 430907) (all from Biolegend) and CCL5 (R\&D, Cat\# DY478) were measured using ELISA kits.

\section{ex vivo chemotaxis assays}

Naïve $C D 8^{+} T$ cells were purified from mouse spleen and activated with Dynabeads containing mouse Tactivator CD3 (Biolegend, Cat\# 100301)/CD28 (Biolegend, Cat\# 102101) and recombinant mouse IL2 (Biolegend, Cat\# 714604). And then, the activated $C D 8^{+} T$ cells were seeded in the upper chambers of transwell plates (BD Biosciences) and allowed to migrate for $24 \mathrm{~h}$ towards the lower chamber containing medium with different concentrations of CCL5 protein (Pepro Tech, Cat\# 250-07).

\section{BMDMs preparation}

Bone marrow cells were isolated from femurs and tibias of C57/BL 6 mice. $5 \times 10^{6}$ cells per well in 24-well plates were cultured in RPMI- 1640 medium containing $10 \%$ heated-inactivated fetal bovine serum at $37^{\circ} \mathrm{C}$ in a $5 \% \mathrm{CO}_{2}$ incubator. Bone marrow derived macrophage (BMDM)s were differentiated in the presence of recombinant cytokine M-CSF (20ng/ml, Pepro Tech, Cat\# 315-02). Every 2 days, 50\% of the medium were replaced with fresh culture medium. After 10 days, we harvested adherent cells and used them for BMDM experiments.

\section{PBMCs preparation}


The peripheral blood mononuclear cell (PBMC)s of NSCLC patients were isolated and rested in RPMI1640 medium containing $10 \%$ heated-inactivated fetal bovine serum at $37^{\circ} \mathrm{C}$ in a $5 \% \mathrm{CO}_{2}$ incubator for 6 h. Then, $5 \times 10^{5}$ cells per well in 24-well plates were cultured different doses of tucidinostat as indicated for $24 \mathrm{~h}$.

The cells were stained with the following Abs according to the manufacturer's instructions: CD14 (Cat\# 325604), CD11b (Cat\# 393112), CD3 (Cat\# 344804), CD4 (Cat\# 317428), CD8a (Cat\# 344722), CD69 (Cat\# 310906), CD86 (Cat\# 374208), or HLA-DR (Cat\# 307630) (2.5ul, all from BioLegend) were diluted in FACS buffer (Biolegend). Data was collected and analyzed with the Flow cytometers (BD Biosciences) and the Flow Jo software (Ashland, OR, USA).

Various immune cells were separated using a gating strategy based on the expression of known lineage markers for total peripheral blood monocytes $\left(C D 14^{+} C D 11 b^{+}\right)$, active peripheral blood monocytes $\left(\mathrm{CD} 14^{+} \mathrm{CD} 11 \mathrm{~b}^{+} \mathrm{HLA}-\mathrm{DR}{ }^{+} / \mathrm{CD} 14^{+} \mathrm{CD} 11 \mathrm{~b}^{+} \mathrm{CD} 86^{+}\right)$, total T cells $\left(\mathrm{CD} 3^{+}\right), \mathrm{CD} 4^{+} \mathrm{T}$ cells $\left(\mathrm{CD} 3^{+} \mathrm{CD} 4^{+}\right), \mathrm{CD} 8^{+} \mathrm{T}$ cells $\left(\mathrm{CD} 3^{+} \mathrm{CD} 8^{+}\right)$, active $\mathrm{CD} 4^{+} \mathrm{T}$ cells $\left(\mathrm{CD} 3^{+} \mathrm{CD} 4^{+} \mathrm{CD} 69^{+}\right)$, and active $\mathrm{CD} 8^{+} \mathrm{T}$ cells $\left(\mathrm{CD} 3^{+} \mathrm{CD} 8^{+} \mathrm{CD} 69^{+}\right)$.

The study was approved by the Institutional Review Board of National Cancer Center/National Clinical Research Center for Cancer/Cancer Hospital, Chinese Academy of Medical Sciences \& Peking Union Medical College (Permit Number, NCC2020C072).

\section{Statistical analysis}

All data analysis was performed using GraphPad Prism software (version 5.0, GraphPad Software, Inc.). For the comparison among treatment groups in the in vitro and in vivo study, one-way ANOVA was performed. Survival time was defined from the day of tumor cell inoculation until the mice expired naturally or euthanized. Survival curves were drawn using Kaplan-Meier method and compared with logrank test. $P<0.05$ was considered statistically significant. In the Figures, symbols were used as: ${ }^{*}<<0.05$, $\star \star P<0.01, * \star * P<0.001$.

\section{Results}

Optimized dose of tucidinostat inhibits tumor growth and activates the tumor immune microenvironment

The antitumor effect of different doses of tucidinostat $(2.5,5$, and $7.5 \mu \mathrm{M})$ was first evaluated in three different cell lines $-4 \mathrm{~T} 1$ breast cancer cells, LLC lung cancer cells, and CT26 colorectal cells-in vitro and the data revealed that the cell proliferation levels were significantly suppressed and the cell apoptosis levels were increased in the higher-dose groups (Supplementary Fig.1a-b). To determine the optimized dose of tucidinostat, the in vivo activity of tucidinostat was assessed in the CT26 tumor-bearing mice. The mice were gavaged with tucidinostat daily at two subclinical doses $(12.5 \mathrm{mg} / \mathrm{kg}, 25 \mathrm{mg} / \mathrm{kg})$ and one clinical dose $(75 \mathrm{mg} / \mathrm{kg}$ ) after tumor cell inoculation (Fig.1a). The administration of the subclinical doses of tucidinostat induced sustained and modest decrease of tumor growth with tolerable toxicity, and the clinical dose of tucidinostat induced significantly greater tumor growth suppression but with intolerable 
toxicities, such as rapid body weight loss, leucopenia, and lymphopenia (Fig.1b-e). The clinical dose of tucidinostat also elevated the serum alanine transaminase level, which indicated that the clinical dose of tucidinostat could affect liver function. To evaluate kidney function, blood urea nitrogen levels were measured; less damage was recorded in these groups (Supplementary Fig.1c).

Immunological changes occurring in TME after tucidinostat treatment were further assessed. Flow cytometry data demonstrated a significant increase in the number of $\mathrm{CD} 8^{+} \mathrm{T}$ cells infiltrating tumors at 10 days postadministration in the tucidinostat $(25 \mathrm{mg} / \mathrm{kg})$ group, indicating that this dose exerted a robust immune priming effect (Fig. $1 \mathrm{f}$ ). Moreover, the $\mathrm{CD} 8^{+} \mathrm{T}$ cells in the drainage lymph node (dLN)s also increased, although the difference was not statistically significant. Interestingly, the number of $\mathrm{CD} 3^{+}$and $\mathrm{CD}^{+}{ }^{+} \mathrm{T}$ cells increased following tucidinostat $(25 \mathrm{mg} / \mathrm{kg})$ treatment, suggesting that the treatment induced effective antitumor immune responses (Fig.1f). Furthermore, immunofluorescence staining of tumor sections demonstrated that the proportion of $\mathrm{CD} 8^{+} \mathrm{T}$ cells was higher in this group (Fig. $1 \mathrm{~g}$ ). Considering the antitumor efficacy and safety profile, the subclinical dose $(25 \mathrm{mg} / \mathrm{kg})$ of tucidinostat was selected for further investigations.

\section{Tucidinostat induces the expression of effector T cell-attracting chemokines and PD-L1}

Next, potential mechanisms underlying the changes in TME were explored through gene expression profiling. Bulk mRNA-seq data comparing tucidinostat-treated $(25 \mathrm{mg} / \mathrm{kg})$ and untreated tumors from the LLC murine model indicated that tucidinostat could markedly alter the TME, as indicated by the significantly higher immune microenvironment scores. A heatmap of differential expressed genes revealed a consistency between immune-related gene signatures and the changes observed in the $T$ cell populations (Fig.2a-b). Furthermore, the functional annotation of gene clustering indicated altered expression levels of a considerable amount of cytokines following tucidinostat treatment (Supplementary Fig.2a).

It is known that the best predictors of immunotherapy response are the number and phenotype of tumorinfiltrating $\mathrm{CD}^{+} \mathrm{T}$ cells recruited at the tumor site by the locally secreted chemokines[34, 37]. A large body of evidence exists to show that increased expression of $C D 8^{+} T$ cell-attracting chemokines, such as the C-C motif chemokine ligand 5 (CCL5) and C-X-C motif chemokine ligand 9 and 10 (CXCL9 \& CXCL10), correlates with decreased levels of cancer metastasis and improved clinical outcome in patients with cancer[38-40]. Recently, a mechanistic link between epigenetic modification and the secretion of such cytokines in TME has been described[41, 42]. Here, we hypothesized that the immune modulation of tucidinostat may occur, at least partially, through tumor-derived cytokine secretion. qPCR were performed for the treated murine 4T1, LLC, and CT26 cancer cell lines, and the data showed that the total expression levels of CCL5, CXCL9, and CXCL10 were significantly increased after tucidinostat treatment (Fig.2C). More importantly, the optimized dose of tucidinostat treatment also improved the total and cell-surface expressions of PD-L1 (Fig.2c, Supplementary Fig.2b). In general, tucidinostat at the optimized dose could elevate the expression of PD-L1 and T cell-attracting chemokines, such as CCL5, CXCL9, and CXCL10. 
Considering that the stress-activated NF-KB pathway controls cytokine expression in multiple cell types and the key role of CCL5 in attracting $\mathrm{CD}^{+} \mathrm{T}$ cells $[43,44]$, we further hypothesized that the antitumor immune response induced by tucidinostat may be mediated through CCL5 upregulation via the NF-KB pathway. As expected, NF-KB pathway was activated along with an increased expression of CCL5 after the administration of tucidinostat treatment in CT26 cancer cell lines, and this upregulation of CCL5 expression was abrogated upon pharmacological NF-KB inhibition using BAY11-7082 (Fig.2d-e).

Previous data suggested that high intratumoral expression of $\mathrm{CD} 8^{+} \mathrm{T}$ cell-attracting chemokine CCL5 is correlated with better prognosis in several types of cancers[45-48]. Moreover, CD8A expression is significantly correlated with $\mathrm{CD} 8^{+} \mathrm{T}$ cell infiltration and surface proteins that are critical to transduce antigen recognition into immune cell responses[49]. Therefore, the prognostic roles of CCL5 and CD8A were evaluated using public datasets from The Cancer Genome Atlas (TCGA). The analysis revealed that CCL5 expression is positively correlated with CD8A expression in three cancer types (breast invasive carcinoma, lung adenocarcinoma, and colon adenocarcinoma)(Supplementary Fig.2c). Furthermore, both CCL5 $5^{\text {High }}$ and CCL5 $5^{\text {High }} \mathrm{CD} 8 \mathrm{~A}^{\text {High }}$ is negatively correlated with the risk of death or recurrence in breast cancer (Fig.2f, Supplementary Fig.2d), which is consistent with previous data. Ex vivo chemotaxis assays have also shown that higher CCL5 protein concentrations could enhance $\mathrm{CD} 8^{+} \mathrm{T}$ cell transwell migration (Fig.2g), suggesting that CCL5 is necessary for T cell infiltration.

Overall, these findings demonstrated a strong intrinsic anticancer activity of tucidinostat, which was mediated by the enhancement of $\mathrm{CD}^{+} \mathrm{T}$ cell recruitment through CCL 5 upregulation via NF-KB signaling pathway activation.

\section{Tucidinostat enhances M1 polarization of macrophages both in vitro and in vivo}

Next, we examined whether tucidinostat can induce an innate antitumor immune response that leads to effective tumor regression. Several immune-related gene expressions were observed in Raw 264.7 cells and bone marrow-derived macrophage (BMDM)s 24h after the administration of tucidinostat treatment. mRNA expression levels of the M1-like macrophage marker inducible nitric oxide synthase and CD86 increased, whereas the expression of the M2-like macrophage marker arginase- 1 and CD206 decreased with tucidinostat treatment compared to those in the untreated control as per qPCR assessment (Fig.3a). Furthermore, flow cytometry analysis revealed dose-dependent elevations of M1-like macrophage surface marker, MHC-II, on both Raw.264.7 cells and BMDMs (Fig.3b). The tumor-conditioned medium from untreated LLC cell cultures promoted M2 polarization of BMDMs and Raw.264.7 cells, whereas the tumorconditioned medium collected after tucidinostat treatment promoted M1 polarization of BMDMs and Raw.264.7 cells as assessed by qPCR and flow cytometry (Fig.3c-d).

To further investigate the influence of tucidinostat on macrophage polarization in vivo, the immunological changes observed in tumors after administering treatment to CT26 tumor-bearing mice were assessed. Compared with other groups, a significant decrease in the proportion of tumor-associated macrophages among the total viable cells and an increase in the ratio of M1 macrophage were observed 
in the tucidinostat-treated $(25 \mathrm{mg} / \mathrm{kg}$ ) group (Fig.3e). Total macrophages in dLNs also distinctly decreased, although the downward trend did not reach statistical difference (Fig.3e).

\section{Tucidinostat improves the immunotherapeutic efficacy of $\mathrm{ICl}$ and induces a durable response}

Because a robust antitumor immune response was induced by daily administration of $25 \mathrm{mg} / \mathrm{kg}$ tucidinostat, the treatment efficacy of tucidinostat combined with ICls was further evaluated in three murine solid tumor models. Accordingly, tumor-bearing mice were treated with tucidinostat $(25 \mathrm{mg} / \mathrm{kg})$ monotherapy through gavage, aPD-L1 ( $200 \mu \mathrm{g}$, every 3 days) monotherapy by intraperitoneal injection, tucidinostat plus aPD-L1, or vehicle control (Fig.4a, Supplementary Fig.3a). Tumor growth was significantly inhibited in the group treated with combination regimens compared to those treated with either single tucidinostat or vehicle control (Fig.4b-d, Supplementary Fig.3b-c). Notably, the combination of tucidinostat and aPD-L1 resulted in the significantly improved survival compared to vehicle control (Fig.4e). To confirm the treatment efficacy of tucidinostat combined with aPD-L1, the tumor-bearing mice were treated with two different HDAC inhibitor, the pan HDAC inhibitor vorinostat and the selective class Ila inhibitor TMP-195, alone or in combination with aPD-L1. Both the combinations yielded mild-tomoderate reduction of tumor burden. The efficacy was less than that of tucidinostat plus aPD-L1 treatment, although the difference observed between these groups did not reach statistical significance (Supplementary Fig.3d).

To investigate the effect of tucidinostat combined with aPD-L1 on TME, the changes observed in the immune cell populations upon treatment were analyzed. In subcutaneous tumors, the total amount of infiltrated $\mathrm{CD}_{4} 5^{+}$lymphocytes was increased after treatment with the combination therapy (Fig.5a). Furthermore, it induced a significant increase in the proportion of tumor-infiltrating $\mathrm{CD} 4^{+}$and $\mathrm{CD} 8^{+} \mathrm{T}$ cells (Fig.5a). In addition, immunohistochemical assays demonstrated an increase in the number of CD $8^{+}$ $T$ cells in tumor tissues collected from the combination therapy group

(Supplementary Fig.4a). Interestingly, $\mathrm{CD}^{+}$and $\mathrm{CD} 4^{+} \mathrm{T}$ cells from the combination therapy group exhibited a noticeable reduction in the expression of the exhaustion marker PD-1 (Supplementary Fig.4b). Furthermore, the reductions in the proportion of tumor-infiltrating macrophages and increases in that of M1 macrophages were observed in the combination therapy group (Fig.5a). The fluorescence-activated cell sorting (FACS) data of dLNs also confirmed that the antitumor activity and immune function were improved following the combination therapy. The percentages of $\mathrm{CD} 4^{+} \mathrm{T}$ cells significantly increased, whereas those of macrophages declined after treatment with tucidinostat plus aPD-L1 (Fig.5b). Other immune cells such as myeloid-derived suppressor cells (MDSCs), DC cells, and natural killer (NK) cells were not significantly influenced in tumor tissues or dLNs (Fig.5a-b, Supplementary Fig.4c).

Furthermore, tucidinostat-induced changes in serum cytokine levels were also examined. In accordance with the changes observed in intratumoral expression of $\mathrm{CD}^{+} \mathrm{T}$ cells, CCL5 level was markedly increased in tumors after tucidinostat treatment alone as well as by the combination of tucidinostat plus aPD-L1 (Supplementary Fig.5a-b). In addition, serum interferon-y (IFN-y) was enhanced in the combination 
therapy group compared with the vehicle control group. However, no changes were observed in serum IL10 or tumor necrosis factor-a (TNF-a) (Supplementary Fig.5c).

In general, these findings indicate that tucidinostat might significantly augment the antitumor immune response of aPD-L1 through $\mathrm{CD}^{+} \mathrm{T}$ cell infiltration and M1 macrophage polarization in solid tumorbearing mice.

\section{$\mathrm{CD}^{+} \mathrm{T}$ cells are required for the induction of antitumor immunity by tucidinostat and aPD-L1 blockade}

As tucidinostat plus PD-L1 blockade resulted in tumor regression and flow cytometry profiling revealed enhanced $\mathrm{CD} 8^{+} \mathrm{T}$ cells infiltration, we hypothesized that the observed antitumor responses were mediated through $\mathrm{CD} 8^{+} \mathrm{T}$ cells populations. To further verify the contribution of $\mathrm{CD} 8^{+} \mathrm{T}$ cells to the enhanced antitumor efficacy of aPD-L1, we developed an in vivo CT26 mouse tumor model and utilized it for examining tumor responses following vehicle control, tucidinostat monotherapy $\pm \mathrm{CD} 8^{+} \mathrm{T}$ cell depletion, and tucidinostat plus aPD-L1 combination therapy $\pm \mathrm{CD} 8^{+} \mathrm{T}$ cell depletion. Upon pretreatment with an anti-CD8 antibody (aCD8), the degree of tumor shrinkage induced by both monotherapy and combination therapy was significant (Fig.6a-d), supporting that tucidinostat potentiated the effects of $\mathrm{PD}-\mathrm{L} 1$ blockade in vivo through $\mathrm{CD} 8^{+} \mathrm{T}$ cell-induced antitumor immune response.

In addition, macrophage depletion using clodronate liposomes also reversed the beneficial effects of combination treatment, suggesting that macrophages also contribute to tumor regression. However, macrophage depletion failed to reverse the antitumor response induced by tucidinostat alone (Fig.6a-d). Therefore, macrophages might be necessary but not sufficient for reversing the tumor suppression effect of tucidinostat.

\section{Tucidinostat increases the expression of the costimulatory molecules on human monocytes but fails to directly promote the transient activation of peripheral $\mathrm{T}$ cells}

Previous studies have reported that tucidinostat could alter antigen-presenting cell (APC) function by regulating inflammatory cytokine production in patients with immune thrombocytopenia[28]. We therefore explored whether tucidinostat could alter the antigen-presenting function of monocytes $\left(\mathrm{CD} 14^{+} \mathrm{CD} 11 \mathrm{~b}^{+}\right)$among the peripheral blood mononuclear cells (PBMCs) of patients with non-small cell lung cancer. After 24h treatment with tucidinostat, the surface expression of CD86 and HLA-DR on the monocyte fraction were significantly upregulated (Fig.7a). These findings demonstrated the modulatory effects of tucidinostat on human monocytes and suggested that tucidinostat promotes these phenotypic changes, conferring enhanced antigen presentation and costimulatory capabilities. However, tucidinostat did not appear to activate T cells directly as no upregulation of the CD69 expression was observed on the conventional $\mathrm{CD}^{+}$or $\mathrm{CD} 4^{+} \mathrm{T}$ cells among PBMCs of patients with non-small cell lung cancer (Fig. $\left.7 \mathrm{~b}\right)$.

\section{Discussion}


HDACs consist of a large family of proteins categorized into five groups-class I (HDAC 1, 2, 3, 8), class Ila (HDAC 4, 5, 7, 9), class IIb (HDAC 6, 10), class III (Sirtuins), and class IV (HDAC 11)[18]. Aberrant HDAC expression occurs in most solid tumors and hematological cancers. The dysregulation of histone acetylation can lead to aberrant gene expression, which can activate oncogenes, inactivate tumor suppressors, inhibit programed cell death, and mediate immune evasion, ultimately resulting in tumor progression[4, 16, 18, 21]. To date, four HDAC inhibitors, vorinostat, romidepsin, panobinostat, and belinostat, have been approved by the United States Food and Drug Administration and are used for treating hematological cancers[50]. Recently, tucidinostat has been approved by the National Medical Products Administration and is used for patients with advanced, hormone receptor-positive, HER2negative breast cancer that progressed after previous endocrine therapy[29]. It might be the first HDAC inhibitor used for treating solid tumors in clinical settings. However, due to the grade 3 or 4 toxicities caused by it, the clinical dose of tucidinostat might not effectively modulate TME. Therefore, the appropriate dose of tucidinostat should been defined for treating solid tumors. In a previous clinical trial involving humans, $30 \mathrm{mg}$ of tucidinostat was orally administered twice a week. After translating the drug dosage from humans to mice, we set the daily clinical dose of tucidinostat in mice as $75 \mathrm{mg} / \mathrm{kg}$ [51]. The mice were gavaged with tucidinostat daily at subclinical doses $(12.5 \mathrm{mg} / \mathrm{kg}, 25 \mathrm{mg} / \mathrm{kg})$ and a clinical dose $(75 \mathrm{mg} / \mathrm{kg})$ after tumor cell inoculation. We demonstrated that tucidinostat at $25 \mathrm{mg} / \mathrm{kg}$ daily dosage could promote a rapid and sustained antitumor immune response through the preclinical mouse tumor model. Conversely, lower or opposite immunosuppressive effects were observed with the administration of lower $(12.5 \mathrm{mg} / \mathrm{kg})$ or clinical dosages $(75 \mathrm{mg} / \mathrm{kg})$. To date, few studies have reported regarding tucidinostat treatment optimization strategies for turning the TME of solid tumors from cold to hot. In this work, we mainly focused on exploring such optimization strategies and then delineating the possible underlying functional mechanisms both in vitro and in vivo.

Next, we demonstrated that the optimized dose tucidinostat could promote the secretion of several CD $8^{+}$ T cell-attracting chemokines, especially that of CCL5. Although the role of CCL5/CCR5 axis in carcinogenesis is controversial[52], increasing evidence has demonstrated that constitutive CCL5 expression enables tumor immune recognition and enhances immunotherapy response via increased infiltration of $\mathrm{CD}^{+} \mathrm{T}$ cells into tumors[53-56]. Besides, high intratumoral expression of CCL5 is correlated with better prognosis and strongly correlated with intratumoral CD8A expression across multiple cancer types according to our analysis of TCGA datasets. It was reported that DNA methylation negatively regulates CCL5 expression in lung and colon cancers[57]. Our findings indicated an additional epigenetic mechanism wherein the selective histone acetylation inhibitor tucidinostat could also induce CCL5 expression in tumors through the NF-KB signaling pathway, leading to CD8+ T cell infiltration into tumors. Because tucidinostat elevated the secretion of CCL5 and other T cell-attracting chemokines in TME, we sought to demonstrate that the optimized dose of tucidinostat can promote a rapid and sustained antitumor immune response when used in combination with aPD-L1 using multiple preclinical mouse tumor models. The response was dependent on enhanced $\mathrm{CD} 8^{+} \mathrm{T}$ cell infiltration in TME and was abrogated upon $\mathrm{CD}^{+} \mathrm{T}$ cell depletion. Thus, tucidinostat with an optimized dose could alter TME and promote the migration and infiltration of $\mathrm{CD} 8^{+} \mathrm{T}$ cells into tumors, partially by increasing the activity of 
chemokine CCL5 via NF-kB signaling. CCL5 might indeed be secreted by some other inflammatory cells in TME following tucidinostat treatment, which is required to be testified in further studies.

Furthermore, we demonstrated that tucidinostat could also modulate M1 polarization of macrophages in solid tumors. It has been reported that the class Ila HDAC inhibitor TMP195 could alter tumor microenvironment and reduce tumor burden and pulmonary metastases by modulating macrophage phenotype in a macrophage-dependent autochthonous mouse model of breast cancer. Furthermore, TMP195 induced the recruitment and differentiation of highly phagocytic and stimulatory macrophages within tumors[58]. In this study, it was seen that tucidinostat could directly promote M1 polarization of macrophages; moreover, the medium from tumor cells treated with tucidinostat could also induce M1 polarization of macrophages, suggesting that factors secreted by tumor cells in response to tucidinostat treatment could repolarize tumor-associated macrophages. The M1 macrophages, which have a role in mediating the destruction of tumor cells and facilitating the recruitment of Th1 cells, were also found to be highly sensitive to tucidinostat plus aPD-L1 treatment. Moreover, the antitumor effect was mostly abolished by macrophage depletion using clodronate liposomes. Therefore, tucidinostat could significantly promote M1 polarization of macrophages and increase the antitumor efficacy of aPD-L1 in vivo.

In addition, significant upregulation of MHC class-II molecules, CD86 and HLA-DR, was observed with phenotypic changes associated with increased APC priming. This observation was consistent with the known positive correlation between MHC class-II expression and PD-1/PD-L1 inhibitor response[59, 60]. In fact, several studies have identified MHC class-II expression as a potential biomarker for PD-1/PD-L1 therapeutic response. Therefore, tucidinostat may sensitize tumors against aPD-1/aPD-L1 blockade, at least partly, by modulating the expression of MHC class-II molecules. However, tucidinostat failed to directly promote the transient activation of peripheral T lymphocytes, which is in agreement with the findings we obtained using mouse models wherein tucidinostat altered T cell function by upregulating T cell-attracting chemokines, such as CCL5.

In summary, the optimized dose of tucidinostat was seen to alter TME by promoting the infiltration of $T$ cells via the activation of the NF-KB pathway and the subsequent release of immune-related cytokines such as CCL5. Moreover, the optimized dose of tucidinostat modulated M1 polarization of macrophages and dramatically potentiated the antitumor efficacy of PD-L1 blockade in solid tumors. Collectively, these findings provide a strong rationale for conducting clinical trials to investigate the combined use of this selective HDAC inhibitor at an optimized dose and PD-L1 blockade for overcoming ICI treatment resistance and achieving better clinical outcomes for patients with solid tumors.

\section{Abbreviations}

ICl, checkpoint inhibitor; HDAC, histone deacetylase; TME, tumor microenvironment; aPD-L1, antiprogrammed cell death ligand 1 antibody; mAb, monoclonal antibody; CCL5, C-C motif chemokine ligand 5; ALT, alanine transaminase; AST, aspartate aminotransferase; $B U N$, urea nitrogen; dLN, drainage lymph 
node; BRCA, breast invasive carcinoma; LUAD, lung adenocarcinoma; COAD, colon adenocarcinoma; PBMC, peripheral blood mononuclear cell; BMDM, bone marrow-derived macrophages; MDSCs, myeloidderived suppressor cells; APC, antigen-presenting cell; iNOS, inducible nitric oxide synthase; Arg 1, arginase 1 ; IFN- $\gamma$, interferon- $\gamma$; TNF-a, tumor necrosis factor-a.

\section{Declarations}

Ethics approval and consent to participate: Animal studies were conducted in accordance with the NIH animal use guidelines and current Chinese regulations and standards for laboratory animal use. The study about PBMC from lung cancer patients was approved by the Institutional Review Board of National Cancer Center/National Clinical Research Center for Cancer/Cancer Hospital, Chinese Academy of Medical Sciences \& Peking Union Medical College.

Consent for publication: Not applicable.

Availability of data and material: All data relevant to the study are included in the article or uploaded as supplemental information.

Competing interests: The authors declare no competing interests.

Funding: This work was supported by the National key research and development project (2019YFC1315700); the National Natural Sciences Foundation Key Program (81630071); National Natural Science Foundation of China (8197112364, 62027901, 81871514, 81227901, 81470083, 91859119, 61671449, 81527805, 61901472); Beijing Natural Science Foundation (7212207), National Public Welfare Basic Scientific Research Program of Chinese Academy of Medical Sciences (2018PT32003, 2017PT32004); National Key R\&D Program of China (018YFC0910602, 2017YFA0205200, 2017YFA0700401, 2016YFA0100902, 2016YFC0103702); Aiyou Foundation (KY201701).

Authors contributions: Jie Wang and Jie Tian supervised the study and were responsible for the study conception and design. Pei Zhang, Xin Wang and Jia Zhong performed the in vivo studies. Rui Wan and Jiachen Xu carried out the immunohistochemistry experiments. Pei Zhang and Xiran He performed the ELISA analysis. Pei Zhang optimized and supervised the flow cytometry experiments. Xiran $\mathrm{He}$ and Wang Di carried out the mRNA and protein expression analyses. Ruofei Yu and Kailun Fei provided clinical samples. Pei Zhang, Yang Du and Hua Bai were responsible for the analysis and interpretation of the data. Zhijie Wang and Jianchun Duan performed the statistical analysis. Pei Zhang and Yang Du wrote the manuscript. All authors critically reviewed the manuscript and approved its final version.

Acknowledgements: The authors would like to acknowledge the instrumental and technical support of Peking Union Medical College and Chinese Academy of Sciences.

\section{References}


1. Freeman GJ, Long AJ, Iwai Y, Bourque K, Chernova T, Nishimura H, Fitz LJ, Malenkovich N, Okazaki T, Byrne MC, Horton HF, Fouser L, Carter L, Ling V, Bowman MR, Carreno BM, Collins M, Wood CR, Honjo T. Engagement of the PD-1 immunoinhibitory receptor by a novel $B 7$ family member leads to negative regulation of lymphocyte activation. J EXP MED 2000;192:1027-1034.

2. Keir ME, Liang SC, Guleria I, Latchman YE, Qipo A, Albacker LA, Koulmanda M, Freeman GJ, Sayegh $\mathrm{MH}$, Sharpe AH. Tissue expression of PD-L1 mediates peripheral T cell tolerance. J EXP MED 2006;203:883-895.

3. Ribas A, Wolchok JD. Cancer immunotherapy using checkpoint blockade. SCIENCE 2018;359:13501355.

4. Chen DS, Mellman I. Elements of cancer immunity and the cancer-immune set point. NATURE 2017;541:321-330.

5. Boussiotis VA. Molecular and Biochemical Aspects of the PD-1 Checkpoint Pathway. N Engl J Med 2016;375:1767-1778.

6. Pitt JM, Vetizou M, Daillere R, Roberti MP, Yamazaki T, Routy B, Lepage P, Boneca IG, Chamaillard M, Kroemer G, Zitvogel L. Resistance Mechanisms to Immune-Checkpoint Blockade in Cancer: TumorIntrinsic and -Extrinsic Factors. IMMUNITY 2016;44:1255-1269.

7. Johnson DB, Sosman JA. Therapeutic Advances and Treatment Options in Metastatic Melanoma. JAMA ONCOL 2015;1:380-386.

8. Patel SA, Minn AJ. Combination Cancer Therapy with Immune Checkpoint Blockade: Mechanisms and Strategies. IMMUNITY 2018;48:417-433.

9. Goel S, DeCristo MJ, Watt AC, BrinJones H, Sceneay J, Li BB, Khan N, Ubellacker JM, Xie S, MetzgerFilho O, Hoog J, Ellis MJ, Ma CX, Ramm S, Krop IE, Winer EP, Roberts TM, Kim HJ, McAllister SS, Zhao JJ. CDK4/6 inhibition triggers anti-tumour immunity. NATURE 2017;548:471-475.

10. Galluzzi L, Buque A, Kepp O, Zitvogel L, Kroemer G. Immunological Effects of Conventional Chemotherapy and Targeted Anticancer Agents. CANCER CELL 2015;28:690-714.

11. Roulois D, Loo YH, Singhania R, Wang Y, Danesh A, Shen SY, Han H, Liang G, Jones PA, Pugh TJ, O'Brien C, De Carvalho DD. DNA-Demethylating Agents Target Colorectal Cancer Cells by Inducing Viral Mimicry by Endogenous Transcripts. CELL 2015;162:961-973.

12. Topper MJ, Vaz M, Chiappinelli KB, DeStefano SC, Niknafs N, Yen RC, Wenzel A, Hicks J, Ballew M, Stone M, Tran PT, Zahnow CA, Hellmann MD, Anagnostou V, Strissel PL, Strick R, Velculescu VE, Baylin SB. Epigenetic Therapy Ties MYC Depletion to Reversing Immune Evasion and Treating Lung Cancer. CELL 2017;171:1284-1300.

13. Chiappinelli KB, Strissel PL, Desrichard A, Li H, Henke C, Akman B, Hein A, Rote NS, Cope LM, Snyder A, Makarov V, Budhu S, Slamon DJ, Wolchok JD, Pardoll DM, Beckmann MW, Zahnow CA, Merghoub T, Chan TA, Baylin SB, Strick R. Inhibiting DNA Methylation Causes an Interferon Response in Cancer via dsRNA Including Endogenous Retroviruses. CELL 2015;162:974-986.

14. Esteller M. Cancer epigenomics: DNA methylomes and histone-modification maps. NAT REV GENET 2007;8:286-298. 
15. Jones PA, Taylor SM. Cellular differentiation, cytidine analogs and DNA methylation. CELL 1980;20:85-93.

16. Potaczek DP, Harb H, Michel S, Alhamwe BA, Renz H, Tost J. Epigenetics and allergy: from basic mechanisms to clinical applications. EPIGENOMICS-UK 2017;9:539-571.

17. Tarakhovsky A. Tools and landscapes of epigenetics. NAT IMMUNOL 2010;11:565-568.

18. Falkenberg KJ, Johnstone RW. Histone deacetylases and their inhibitors in cancer, neurological diseases and immune disorders. NAT REV DRUG DISCOV 2014;13:673-691.

19. Mohammad HP, Barbash O, Creasy CL. Targeting epigenetic modifications in cancer therapy: erasing the roadmap to cancer. NAT MED 2019;25:403-418.

20. Valdez BC, Li Y, Murray D, Brammer JE, Liu Y, Hosing C, Nieto Y, Champlin RE, Andersson BS. Differential effects of histone deacetylase inhibitors on cellular drug transporters and their implications for using epigenetic modifiers in combination chemotherapy. Oncotarget 2016;7:6382963838.

21. Popovic R, Licht JD. Emerging epigenetic targets and therapies in cancer medicine. CANCER DISCOV 2012;2:405-413.

22. Hui KF, Chiang AK. Combination of proteasome and class I HDAC inhibitors induces apoptosis of NPC cells through an HDAC6-independent ER stress-induced mechanism. INT J CANCER 2014;135:2950-2961.

23. Ny L, Jespersen H, Karlsson J, Alsen S, Filges S, All-Eriksson C, Andersson B, Carneiro A, Helgadottir H, Levin M, Ljuslinder I, Olofsson BR, Sah VR, Stierner U, Stahlberg A, Ullenhag G, Nilsson LM, Nilsson JA. The PEMDAC phase 2 study of pembrolizumab and entinostat in patients with metastatic uveal melanoma. NAT COMMUN 2021;12:5155.

24. Baretti M, Yarchoan M. Epigenetic modifiers synergize with immune-checkpoint blockade to enhance long-lasting antitumor efficacy. J CLIN INVEST 2021;131.

25. Ning ZQ, Li ZB, Newman MJ, Shan S, Wang XH, Pan DS, Zhang J, Dong M, Du X, Lu XP. Chidamide (CS055/HBI-8000): a new histone deacetylase inhibitor of the benzamide class with antitumor activity and the ability to enhance immune cell-mediated tumor cell cytotoxicity. Cancer Chemother Pharmacol 2012;69:901-909.

26. Shi Y, Dong M, Hong X, Zhang W, Feng J, Zhu J, Yu L, Ke X, Huang H, Shen Z, Fan Y, Li W, Zhao X, Qi J, Huang H, Zhou D, Ning Z, Lu X. Results from a multicenter, open-label, pivotal phase II study of chidamide in relapsed or refractory peripheral T-cell lymphoma. ANN ONCOL 2015;26:1766-1771.

27. Shen L, Pili R. Class I histone deacetylase inhibition is a novel mechanism to target regulatory $T$ cells in immunotherapy. ONCOIMMUNOLOGY 2012;1:948-950.

28. Zhao HY, Ma YH, Li DQ, Sun T, Li LZ, Li P, Liu XG, Zhou H, Hou Y, Liu Y, Han PP, Zhao YJ, Jing FM, Peng J, Hou M. Low-dose chidamide restores immune tolerance in ITP in mice and humans. BLOOD 2019;133:730-742.

29. Jiang Z, Li W, Hu X, Zhang Q, Sun T, Cui S, Wang S, Ouyang Q, Yin Y, Geng C, Tong Z, Cheng Y, Pan Y, Sun Y, Wang H, Ouyang T, Gu K, Feng J, Wang X, Wang S, Liu T, Gao J, Cristofanilli M, Ning Z, Lu X. 
Tucidinostat plus exemestane for postmenopausal patients with advanced, hormone receptorpositive breast cancer (ACE): a randomised, double-blind, placebo-controlled, phase 3 trial. LANCET ONCOL 2019;20:806-815.

30. Pfirschke C, Engblom C, Rickelt S, Cortez-Retamozo V, Garris C, Pucci F, Yamazaki T, Poirier-Colame V, Newton A, Redouane Y, Lin YJ, Wojtkiewicz G, Iwamoto Y, Mino-Kenudson M, Huynh TG, Hynes RO, Freeman GJ, Kroemer G, Zitvogel L, Weissleder R, Pittet MJ. Immunogenic Chemotherapy Sensitizes Tumors to Checkpoint Blockade Therapy. IMMUNITY 2016;44:343-354.

31. de Biasi AR, Villena-Vargas J, Adusumilli PS. Cisplatin-induced antitumor immunomodulation: a review of preclinical and clinical evidence. CLIN CANCER RES 2014;20:5384-5391.

32. Okita R, Yukawa T, Nojima Y, Maeda A, Saisho S, Shimizu K, Nakata M. MHC class I chain-related molecule $A$ and $B$ expression is upregulated by cisplatin and associated with good prognosis in patients with non-small cell lung cancer. Cancer Immunol Immunother 2016;65:499-509.

33. He X, Du Y, Wang Z, Wang X, Duan J, Wan R, Xu J, Zhang P, Wang D, Tian Y, Han J, Fei K, Bai H, Tian $\mathrm{J}$, Wang J. Upfront dose-reduced chemotherapy synergizes with immunotherapy to optimize chemoimmunotherapy in squamous cell lung carcinoma. J IMMUNOTHER CANCER 2020;8.

34. Reagan-Shaw S, Nihal M, Ahmad N. Dose translation from animal to human studies revisited. FASEB J 2008;22:659-661.

35. Zhang M, Luo JL, Sun Q, Harber J, Dawson AG, Nakas A, Busacca S, Sharkey AJ, Waller D, Sheaff MT, Richards C, Wells-Jordan P, Gaba A, Poile C, Baitei EY, Bzura A, Dzialo J, Jama M, Le Quesne J, Bajaj A, Martinson L, Shaw JA, Pritchard C, Kamata T, Kuse N, Brannan L, De Philip ZP, Yang H, Griffiths G, Wilson G, Swanton C, Dudbridge F, Hollox EJ, Fennell DA. Clonal architecture in mesothelioma is prognostic and shapes the tumour microenvironment. NAT COMMUN 2021;12:1751.

36. Zhang M, Luo JL, Sun Q, Harber J, Dawson AG, Nakas A, Busacca S, Sharkey AJ, Waller D, Sheaff MT, Richards C, Wells-Jordan P, Gaba A, Poile C, Baitei EY, Bzura A, Dzialo J, Jama M, Le Quesne J, Bajaj A, Martinson L, Shaw JA, Pritchard C, Kamata T, Kuse N, Brannan L, De Philip ZP, Yang H, Griffiths G, Wilson G, Swanton C, Dudbridge F, Hollox EJ, Fennell DA. Author Correction: Clonal architecture in mesothelioma is prognostic and shapes the tumour microenvironment. NAT COMMUN 2021;12:3569.

37. Carlino MS, Larkin J, Long GV. Immune checkpoint inhibitors in melanoma. LANCET 2021;398:10021014.

38. Spranger S, Bao R, Gajewski TF. Melanoma-intrinsic beta-catenin signalling prevents anti-tumour immunity. NATURE 2015;523:231-235.

39. Harlin H, Meng Y, Peterson AC, Zha Y, Tretiakova M, Slingluff C, McKee M, Gajewski TF. Chemokine expression in melanoma metastases associated with CD8+ T-cell recruitment. CANCER RES 2009;69:3077-3085.

40. Peng D, Kryczek I, Nagarsheth N, Zhao L, Wei S, Wang W, Sun Y, Zhao E, Vatan L, Szeliga W, Kotarski J, Tarkowski R, Dou Y, Cho K, Hensley-Alford S, Munkarah A, Liu R, Zou W. Epigenetic silencing of 
TH1-type chemokines shapes tumour immunity and immunotherapy. NATURE 2015;527:249-253.

41. Qin Y, Vasilatos SN, Chen L, Wu H, Cao Z, Fu Y, Huang M, Vlad AM, Lu B, Oesterreich S, Davidson NE, Huang Y. Inhibition of histone lysine-specific demethylase 1 elicits breast tumor immunity and enhances antitumor efficacy of immune checkpoint blockade. ONCOGENE 2019;38:390-405.

42. Dangaj D, Bruand M, Grimm AJ, Ronet C, Barras D, Duttagupta PA, Lanitis E, Duraiswamy J, Tanyi JL, Benencia F, Conejo-Garcia J, Ramay HR, Montone KT, Powell DJ, Gimotty PA, Facciabene A, Jackson DG, Weber JS, Rodig SJ, Hodi SF, Kandalaft LE, Irving M, Zhang L, Foukas P, Rusakiewicz S, Delorenzi M, Coukos G. Cooperation between Constitutive and Inducible Chemokines Enables T Cell Engraftment and Immune Attack in Solid Tumors. CANCER CELL 2019;35:885-900.

43. Hirano F, Komura K, Fukawa E, Makino I. Tumor necrosis factor alpha (TNF-alpha)-induced RANTES chemokine expression via activation of NF-kappaB and p38 MAP kinase: roles of TNF-alpha in alcoholic liver diseases. J HEPATOL 2003;38:483-489.

44. Barbie TU, Alexe G, Aref AR, Li S, Zhu Z, Zhang X, Imamura Y, Thai TC, Huang Y, Bowden M, Herndon J, Cohoon TJ, Fleming T, Tamayo P, Mesirov JP, Ogino S, Wong KK, Ellis MJ, Hahn WC, Barbie DA, Gillanders WE. Targeting an IKBKE cytokine network impairs triple-negative breast cancer growth. J CLIN INVEST 2014;124:5411-5423.

45. Harlin H, Meng Y, Peterson AC, Zha Y, Tretiakova M, Slingluff C, McKee M, Gajewski TF. Chemokine expression in melanoma metastases associated with CD8+ T-cell recruitment. CANCER RES 2009;69:3077-3085.

46. Peng D, Kryczek I, Nagarsheth N, Zhao L, Wei S, Wang W, Sun Y, Zhao E, Vatan L, Szeliga W, Kotarski J, Tarkowski R, Dou Y, Cho K, Hensley-Alford S, Munkarah A, Liu R, Zou W. Epigenetic silencing of TH1-type chemokines shapes tumour immunity and immunotherapy. NATURE 2015;527:249-253.

47. Sugiyama E, Togashi Y, Takeuchi Y, Shinya S, Tada Y, Kataoka K, Tane K, Sato E, Ishii G, Goto K, Shintani Y, Okumura M, Tsuboi M, Nishikawa H. Blockade of EGFR improves responsiveness to PD-1 blockade in EGFR-mutated non-small cell lung cancer. Sci Immunol 2020;5.

48. Tang KH, Li S, Khodadadi-Jamayran A, Jen J, Han H, Guidry K, Chen T, Hao Y, Fedele C, Zebala JA, Maeda DY, Christensen JG, Olson P, Athanas A, Loomis CA, Tsirigos A, Wong KK, Neel BG. Combined Inhibition of SHP2 and CXCR1/2 Promotes Anti-Tumor T Cell Response in NSCLC. CANCER DISCOV 2021.

49. Ock CY, Keam B, Kim S, Lee JS, Kim M, Kim TM, Jeon YK, Kim DW, Chung DH, Heo DS. Pan-Cancer Immunogenomic Perspective on the Tumor Microenvironment Based on PD-L1 and CD8 T-Cell Infiltration. CLIN CANCER RES 2016;22:2261-2270.

50. Yoon S, Eom GH. HDAC and HDAC Inhibitor: From Cancer to Cardiovascular Diseases. Chonnam Med J 2016;52:1-11.

51. Reagan-Shaw S, Nihal M, Ahmad N. Dose translation from animal to human studies revisited. FASEB J 2008;22:659-661.

52. Aldinucci D, Borghese C, Casagrande N. The CCL5/CCR5 Axis in Cancer Progression. Cancers (Basel) $2020 ; 12$. 
53. Griss J, Bauer W, Wagner C, Simon M, Chen M, Grabmeier-Pfistershammer K, Maurer-Granofszky M, Roka F, Penz T, Bock C, Zhang G, Herlyn M, Glatz K, Laubli H, Mertz KD, Petzelbauer P, Wiesner T, Hartl M, Pickl WF, Somasundaram R, Steinberger P, Wagner SN. B cells sustain inflammation and predict response to immune checkpoint blockade in human melanoma. NAT COMMUN 2019;10:4186.

54. Ruiz DGM, Bresnahan E, Molina-Sanchez P, Lindblad KE, Maier B, Sia D, Puigvehi M, Miguela V, Casanova-Acebes M, Dhainaut M, Villacorta-Martin C, Singhi AD, Moghe A, von Felden J, Tal GL, Wang S, Kamphorst AO, Monga SP, Brown BD, Villanueva A, Llovet JM, Merad M, Lujambio A. betaCatenin Activation Promotes Immune Escape and Resistance to Anti-PD-1 Therapy in Hepatocellular Carcinoma. CANCER DISCOV 2019;9:1124-1141.

55. Sen T, Della CC, Milutinovic S, Cardnell RJ, Diao L, Ramkumar K, Gay CM, Stewart CA, Fan Y, Shen L, Hansen RJ, Strouse B, Hedrick MP, Hassig CA, Heymach JV, Wang J, Byers LA. Combination Treatment of the Oral CHK1 Inhibitor, SRA737, and Low-Dose Gemcitabine Enhances the Effect of Programmed Death Ligand 1 Blockade by Modulating the Immune Microenvironment in SCLC. J THORAC ONCOL 2019;14:2152-2163.

56. Fu D, Wu J, Lai J, Liu Y, Zhou L, Chen L, Zhang Q. T cell recruitment triggered by optimal dose platinum compounds contributes to the therapeutic efficacy of sequential PD-1 blockade in a mouse model of colon cancer. AM J CANCER RES 2020;10:473-490.

57. Aznar MA, Labiano S, Diaz-Lagares A, Molina C, Garasa S, Azpilikueta A, Etxeberria I, SanchezPaulete AR, Korman AJ, Esteller M, Sandoval J, Melero I. CD137 (4-1BB) Costimulation Modifies DNA Methylation in CD8(+) T Cell-Relevant Genes. CANCER IMMUNOL RES 2018;6:69-78.

58. Guerriero JL, Sotayo A, Ponichtera HE, Castrillon JA, Pourzia AL, Schad S, Johnson SF, Carrasco RD, Lazo S, Bronson RT, Davis SP, Lobera M, Nolan MA, Letai A. Class Ila HDAC inhibition reduces breast tumours and metastases through anti-tumour macrophages. NATURE 2017;543:428-432.

59. Johnson DB, Estrada MV, Salgado R, Sanchez V, Doxie DB, Opalenik SR, Vilgelm AE, Feld E, Johnson AS, Greenplate AR, Sanders ME, Lovly CM, Frederick DT, Kelley MC, Richmond A, Irish JM, Shyr Y, Sullivan RJ, Puzanov I, Sosman JA, Balko JM. Melanoma-specific MHC-Il expression represents a tumour-autonomous phenotype and predicts response to anti-PD-1/PD-L1 therapy. NAT COMMUN 2016;7:10582.

60. Rodig SJ, Gusenleitner D, Jackson DG, Gjini E, Giobbie-Hurder A, Jin C, Chang H, Lovitch SB, Horak C, Weber JS, Weirather JL, Wolchok JD, Postow MA, Pavlick AC, Chesney J, Hodi FS. MHC proteins confer differential sensitivity to CTLA-4 and PD-1 blockade in untreated metastatic melanoma. SCI TRANSL MED 2018;10.

\section{Figures}


a

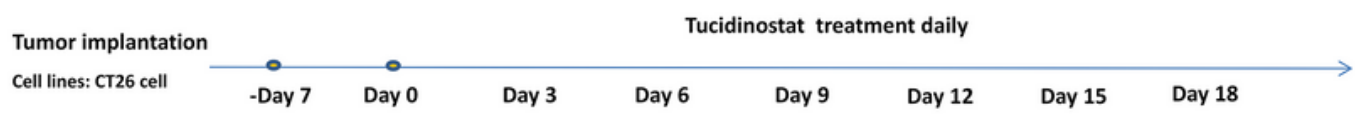

b

c

d
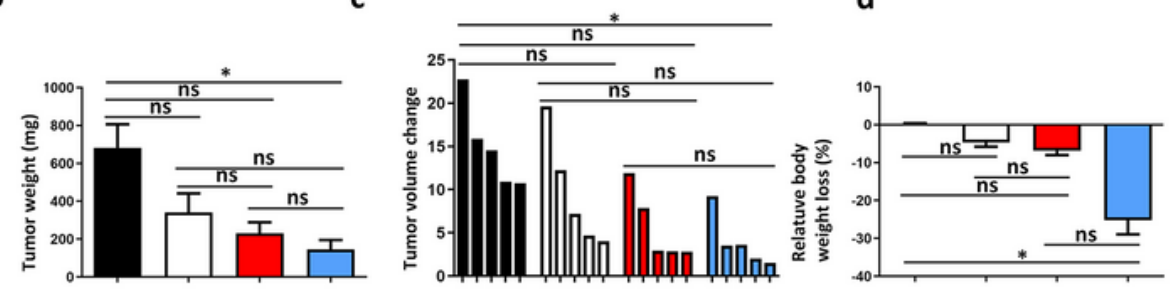

- CON

ㅁ Tuc- $12.5 \mathrm{mg} / \mathrm{kg}$

- Tuc- $25 \mathrm{mg} / \mathrm{kg}$

- Tuc-75 mg/kg

e
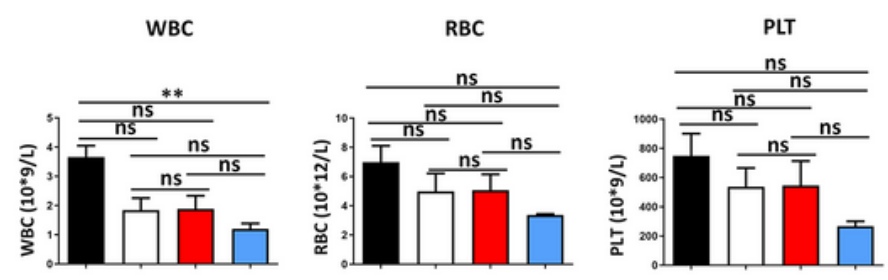

Lymphocytes

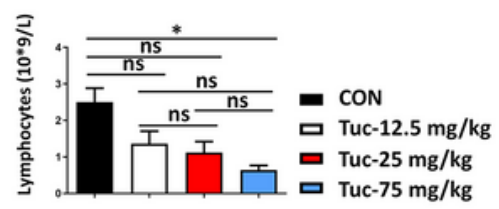

f
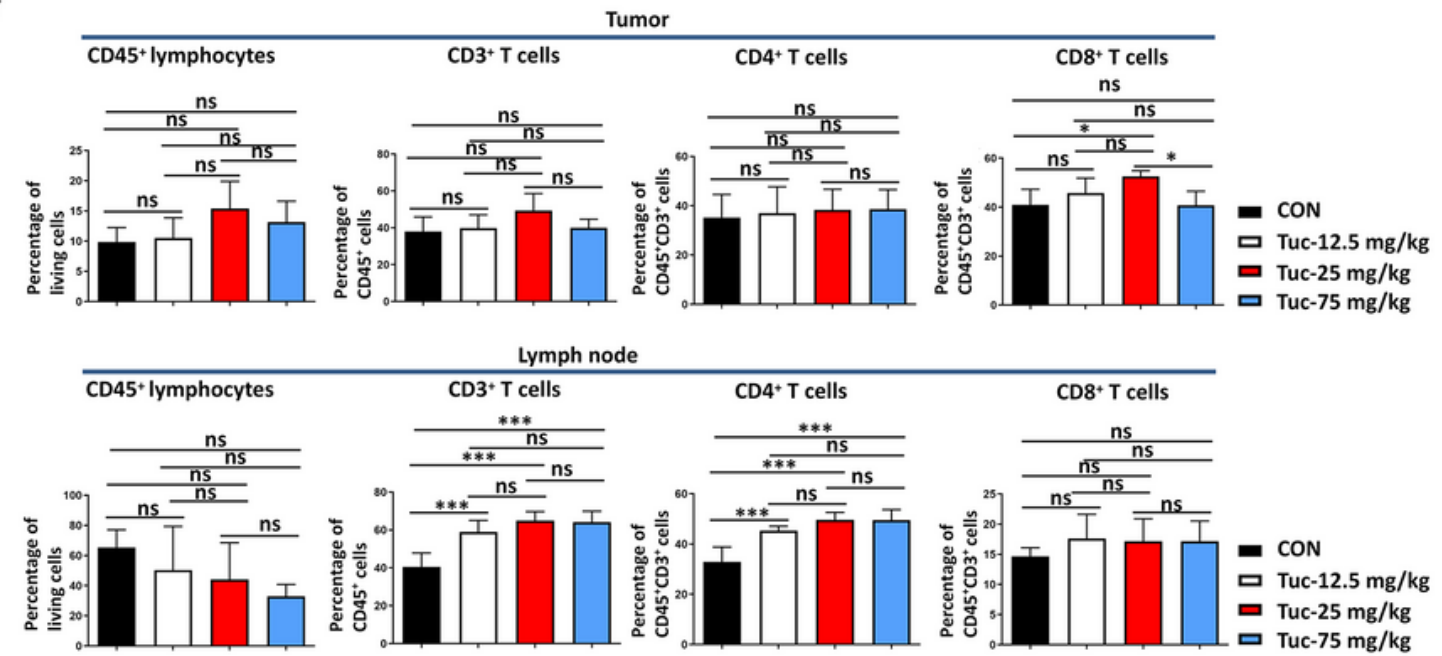

g
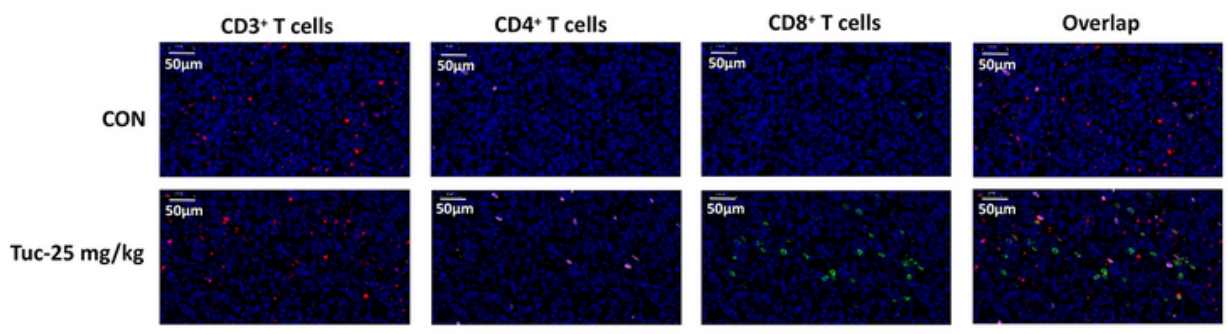

\section{Figure 1}

Optimized dose tucidinostat inhibits tumor growth via modulating antitumor immune response.

(a) Schema of the experiment. Mouse CT26 cells $\left(5 \times 10^{5}\right.$ cells) were engrafted into the flank of BALB/c mice. When established tumors were palpable 7 days after tumor cells inoculation, mice were treated with different doses $(12.5,25,75 \mathrm{mg} / \mathrm{kg}$, gavage, daily, $\mathrm{n}=5)$ of tucidinostat or DMSO as vehicle control 
(DMSO, $n=5$ ). (b) Tumor weight on day 10 post treatment initiation. (c) Waterfall plot of individual tumor volume changes on day 10 post treatment initiation. (d) The percentage of body weight change on day 10 post treatment initiation. (e) Hematological parameters on day 10 post treatment initiation. The blood samples were collected and determined using routine blood test. Routine blood tests include WBC count, RBC count, PLT count, and lymphocytes. (f) Flow cytometric quantification of lymphocytes $\left(C D 45^{+}\right)$, total T cells $\left(\mathrm{CD} 45^{+} \mathrm{CD}^{+}\right), \mathrm{CD} 4^{+} \mathrm{T}$ cells $\left(\mathrm{CD} 45^{+} \mathrm{CD} 3^{+} \mathrm{CD} 4^{+}\right)$, and $\mathrm{CD} 8^{+} \mathrm{T}$ cells $\left(\mathrm{CD} 45^{+} \mathrm{CD} 3^{+} \mathrm{CD} 8^{+}\right)$in tumor parenchyma and tumor drainage lymph nodes from CT26 tumor-bearing mice on day 10 post treatment initiation. (g) Representative immunofluorescent staining for tumor infiltrating T cells on day 10 post treatment initiation. Red, CD3 staining; Pink, CD4 staining; Green, CD8 staining; Blue, DAPI staining.

The error bars indicate \pm SEM. ${ }^{*} \mathrm{P}<0.05,{ }^{*} \mathrm{P}<0.01,{ }^{*} * \mathrm{P}<0.001$ by one-way ANOVA. ns: not significant. WBC: white blood cell; RBC: red blood cell; PLT: platelet; CON: control group; Tuc: tucidinostat. 
Figure 2. Optimized dose tucidinostat promotes CD8+ $\mathrm{T}$ cell migration by inducing CCL5 secretion by tumor cells.

a

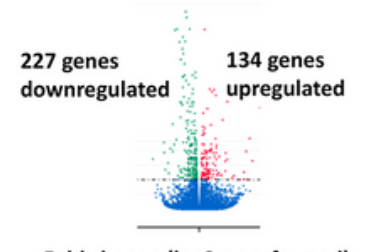

Fold change ( $\log 2$ transformed) b

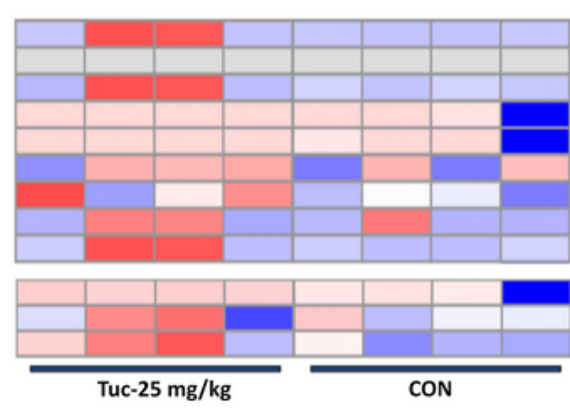

$\mathrm{CD4}^{+}$memory T cells

$\mathrm{CD}^{+}$naive $\mathrm{T}$ cells

$\mathrm{CD}^{+}+\mathrm{T}$ cells

CD4* Tcm

$\mathrm{CD}^{+}{ }^{+} \mathrm{Tem}$

$\mathrm{CD}^{+}$naive $\mathrm{T}$ cells

CD8 $+\mathrm{T}$ cells

$\mathrm{CD} 8{ }^{+} \mathrm{Tcm}$

CD8 ${ }^{+}$Tem

Immune Score

Stroma Score

Microenviroment Score

C

4T1 cells

CT26 cells

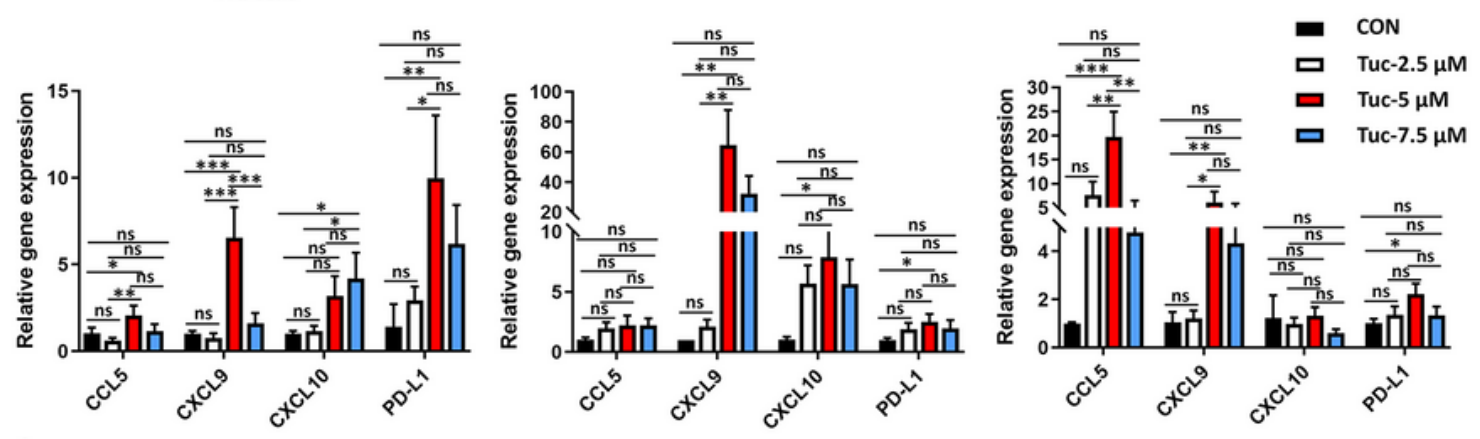

d

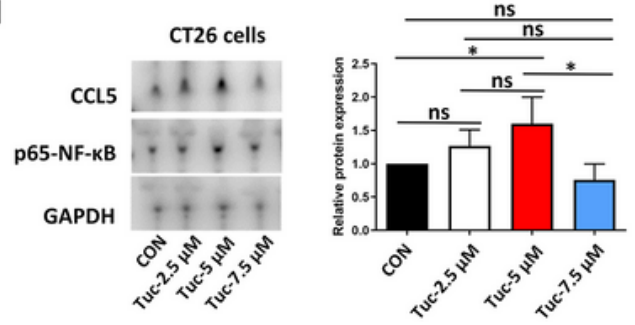

e
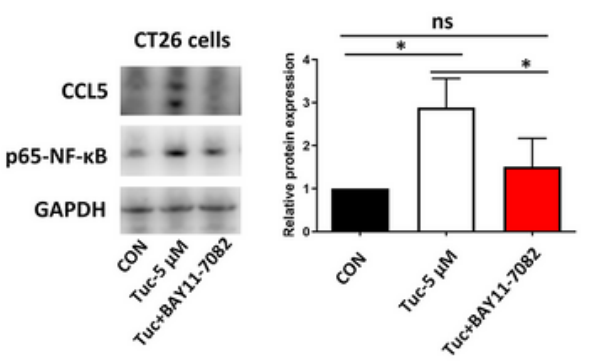

f
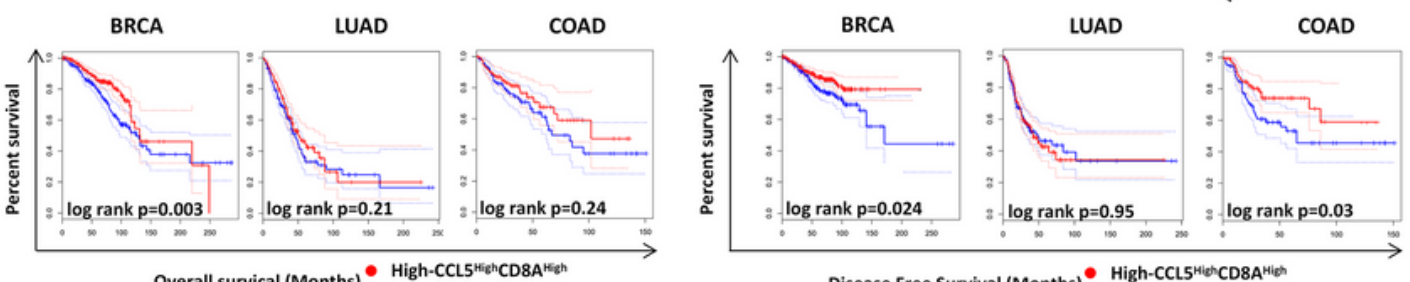

Disease Free Survival (Months) $\bullet$ High-CCL5 HienCD8A Hilen

g
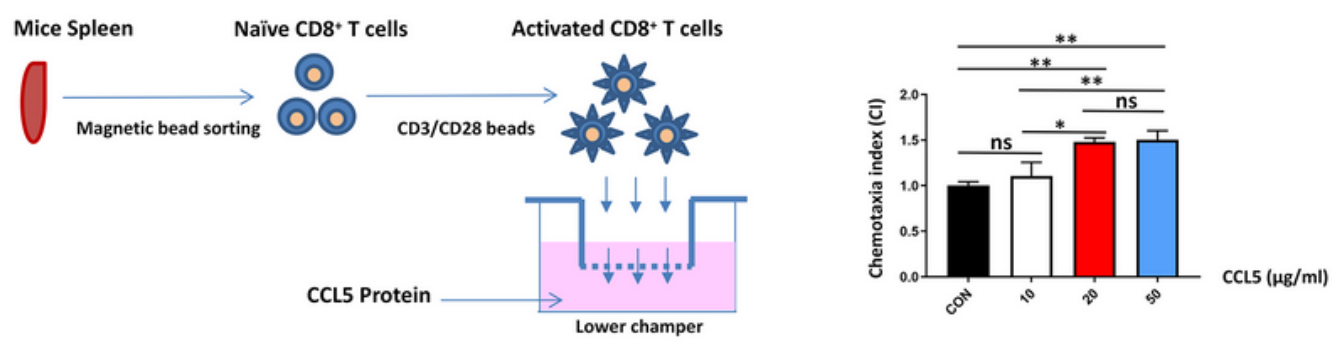

Figure 2

Optimized dose tucidinostat promotes $\mathrm{CD}^{+} \mathrm{T}$ cell migration by inducing pro-inflammatory CCL5 secretion in tumor.

(a) Mouse LLC cells ( $5 \times 10^{5}$ cells) were engrafted into the flank of C57 BL/ 6 mice. When established tumors were palpable 7 days after tumor cells inoculation, mice were treated with tucidinostat $(25 \mathrm{mg} / \mathrm{kg}$, 
gavage, daily, $n=4$ ) or DMSO as vehicle control (DMSO, $n=4)$. Volcano plot showing the significantly overexpressed genes (red) and significant under-expressed genes (green) in tumor from LLC tumor-bearing mice on day 10 post treatment initiation. (b) Heatmap showing the scores of immune gene signatures in tumor from LLC tumor-bearing mice on day 10 post treatment initiation. Colors in the heatmap represent the level of significance of the enrichment (-log10 of the adjusted p values). (c) Relative mRNA expression of CCL5, CXCL9, CXCL10, and PD-L1 compared to vehicle (set to fold change $=1$ ) in 4T1, LLC, and CT26 cells that were exposed to increasing concentrations of tucidinostat $(2.5 \mu \mathrm{M}, 5 \mu \mathrm{M}, 7.5 \mu \mathrm{M})$ for $24 \mathrm{~h}$. The experiment was performed in triplicate. (d) Relative protein expression of CCL5 compared to vehicle in CT26 cells that were exposed to increasing concentrations of tucidinostat $(2.5 \mu \mathrm{M}, 5 \mu \mathrm{M}, 7.5 \mu \mathrm{M})$ for $48 \mathrm{~h}$. The experiment was performed in triplicate. (e) Relative protein expression of CCL5 compared to vehicle in CT26 cells that were exposed to tucidinostat and NF-KB inhibitor (BAY11-7082) for 48h. The experiment was performed in triplicate. (f) Kaplan-Meier survival curves for overall survival and disease free survival in three solid tumor types as stratified by $C C L 5^{\text {High }} \mathrm{CD} 8 \mathrm{~A}^{\mathrm{High}}$ or $\mathrm{CCL} 5^{\text {low }} \mathrm{CD} 8 \mathrm{~A}^{\text {low }}$ expression status using TCGA database. $(\mathrm{g}) \mathrm{CD} 8^{+} \mathrm{T}$ cell migration with different doses of CCL5 protein $(10 \mu \mathrm{g} / \mathrm{ml}$, $20 \mu \mathrm{g} / \mathrm{ml}, 50 \mu \mathrm{g} / \mathrm{ml}$ ) for $24 \mathrm{~h}$. The experiment was performed in triplicate.

The error bars indicate \pm SEM. ${ }^{*} \mathrm{P}<0.05,{ }^{*} \mathrm{P}<0.01,{ }^{\star *} \mathrm{P}<0.001$ by one-way ANOVA. ns: not significant. BRCA: Breast invasive carcinoma; LUAD: Lung adenocarcinoma; COAD: Colon adenocarcinoma; CON: control group; Tuc: tucidinostat. 
Figure 3. Tucidinostat enhances macrophage M1 polarization in vitro and in vivo.

a

Raw 264.7 cells

BMDM
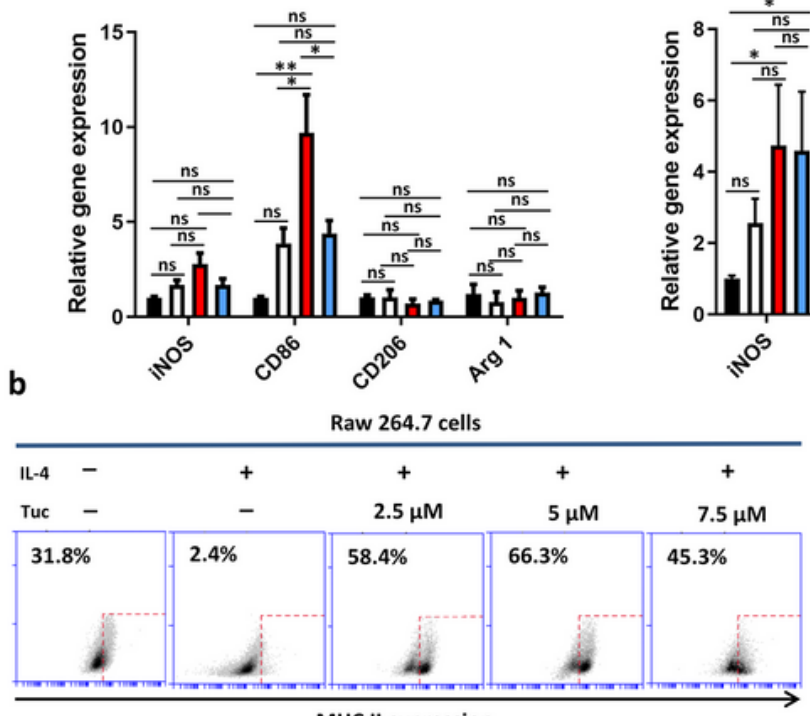

MHC II expression

C

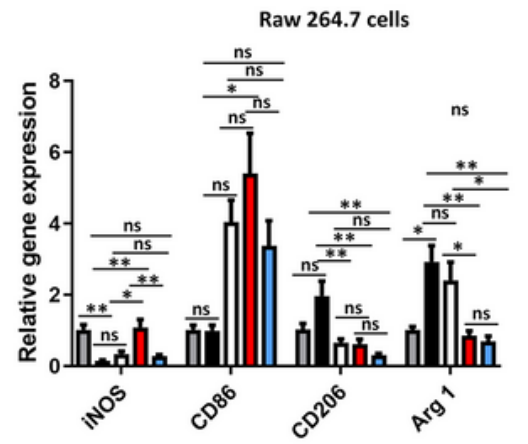

d

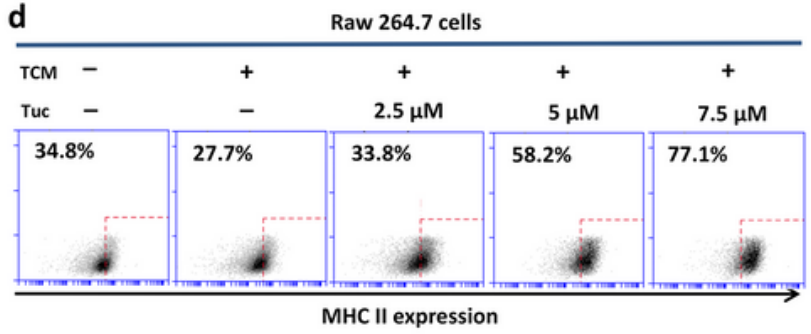

e

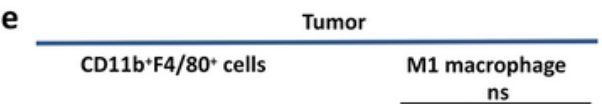

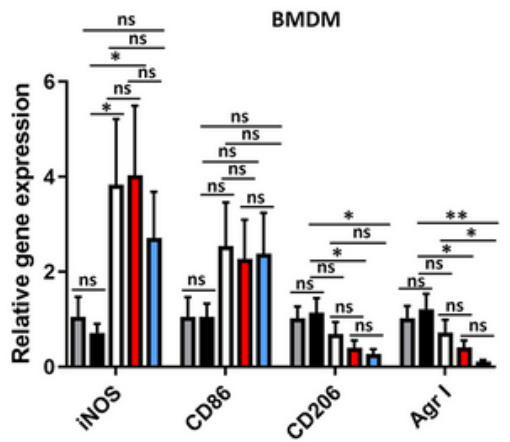

口 CON

- $\mathrm{TCM}+\mathrm{CON}$

口 TCM+Tuc-2.5 $\mu \mathrm{M}$

口 TCM+Tuc-5 $\mu \mathrm{M}$

口 $\mathrm{TCM}+\mathrm{TuC}-7.5 \mu \mathrm{M}$

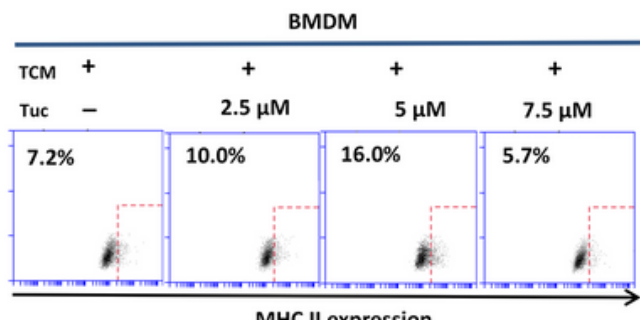

Lymph node

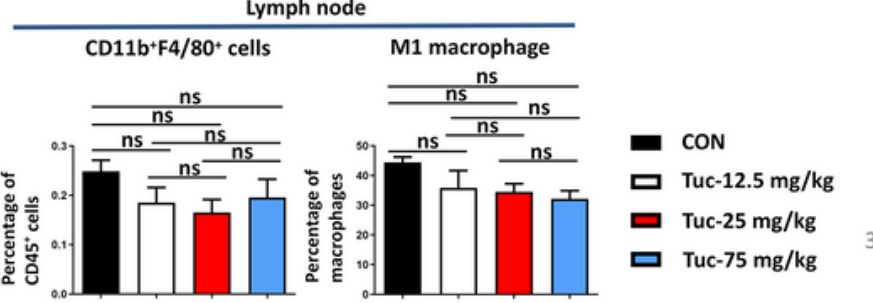

Figure 3

Tucidinostat enhances macrophage M1 polarization in vitro and in vivo.

(a) Relative mRNA expression of iNOS, CD86, CD206, and Arg 1 compared to vehicle (set to fold change $=1$ ) in Raw.264.7 cells and BMDMs that were exposed to increasing concentrations of tucidinostat $(2.5 \mu \mathrm{M}, 5 \mu \mathrm{M}, 7.5 \mu \mathrm{M})$ for $24 \mathrm{~h}$. The experiment was performed in triplicate. (b) Representative cytograms 
for the expression levels of MHC-II on Raw.264.7 cells and BMDMs that were exposed to increasing concentrations of tucidinostat $(2.5 \mu \mathrm{M}, 5 \mu \mathrm{M}, 7.5 \mu \mathrm{M})$ for $24 \mathrm{~h}$. (c) LLC cells being exposed to increasing concentrations of tucidinostat $(2.5 \mu \mathrm{M}, 5 \mu \mathrm{M}, 7.5 \mu \mathrm{M})$ for $24 \mathrm{~h}$ and the tumor conditioned medium being collected and added into Raw.264.7 cells and BMDMs for 24h. Relative mRNA expression of iNOS, CD86, CD206, and Arg 1 compared to vehicle (set to fold change=1) in Raw.264.7 cells and BMDMs that were exposed to such tumor conditioned medium. The experiment was performed in triplicate. (d) Representative cytograms for the expression levels of MHC-II on Raw.264.7 cells and BMDMs that were exposed to such tumor conditioned medium. (e) Mouse CT26 cells ( $5 \times 10^{5}$ cells) were engrafted into the flank of BALB/c mice. When established tumors were palpable 7 days after tumor cells inoculation, mice were treated with different doses $(12.5,25,75 \mathrm{mg} / \mathrm{kg}$, gavage, daily, $\mathrm{n}=5)$ of tucidinostat or DMSO as vehicle control (DMSO, $n=5)$. Percentage of total macrophages $\left(C D 45^{+} C D 11 b^{+} F 4 / 80^{+}\right)$and the percentage of $\mathrm{M} 1$ macrophages $\left(\mathrm{CD} 45^{+} \mathrm{CD} 11 \mathrm{~b}^{+} \mathrm{F} 4 / 80^{+} / \mathrm{MHC}-\mathrm{II}^{+}\right)$in tumor parenchyma and tumor drainage lymph nodes from CT26 tumor-bearing mice on day 10 post treatment initiation.

The error bars indicate \pm SEM. ${ }^{*} \mathrm{P}<0.05,{ }^{*} \mathrm{P}<0.01,{ }^{*} * \mathrm{P}<0.001$ by one-way ANOVA. ns: not significant. BMDM: bone marrow-derived macrophages; CON: control group; TCM: tumor-conditioned medium; Tuc: tucidinostat; iNOS, inducible nitric oxide synthase; Arg 1, arginase-1. 
Figure 4. Tucidinostat improves the efficacy of checkpoint blockade and induces a durable response.

a

\begin{tabular}{|c|c|c|c|c|c|c|c|c|}
\hline \multirow[t]{2}{*}{ Tumor implantation } & \multicolumn{8}{|c|}{ Tuc- $25 \mathrm{mg} / \mathrm{kg}$ treatment daily } \\
\hline & -Day 7 & Day 0 & Day 3 & Day 6 & Day 9 & Day 12 & Day 15 & Day 18 \\
\hline
\end{tabular}
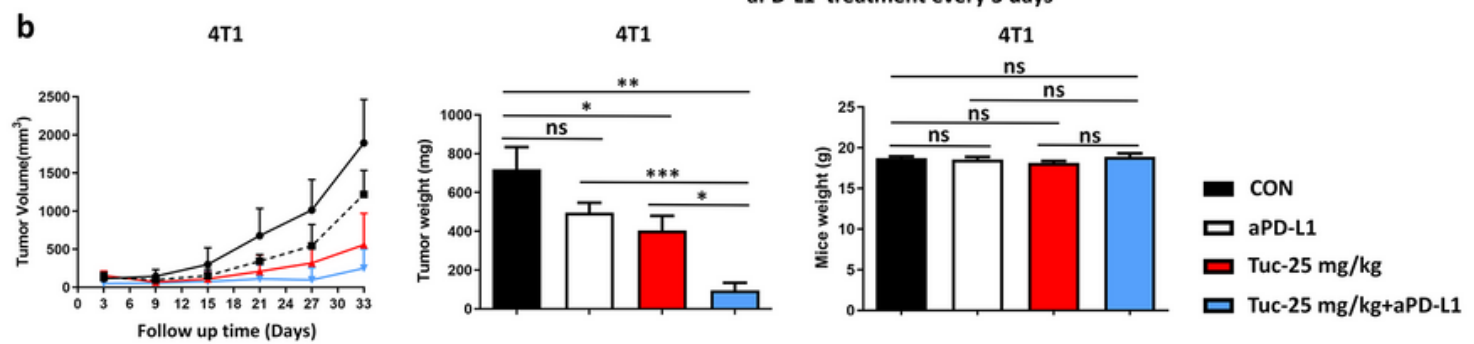

C
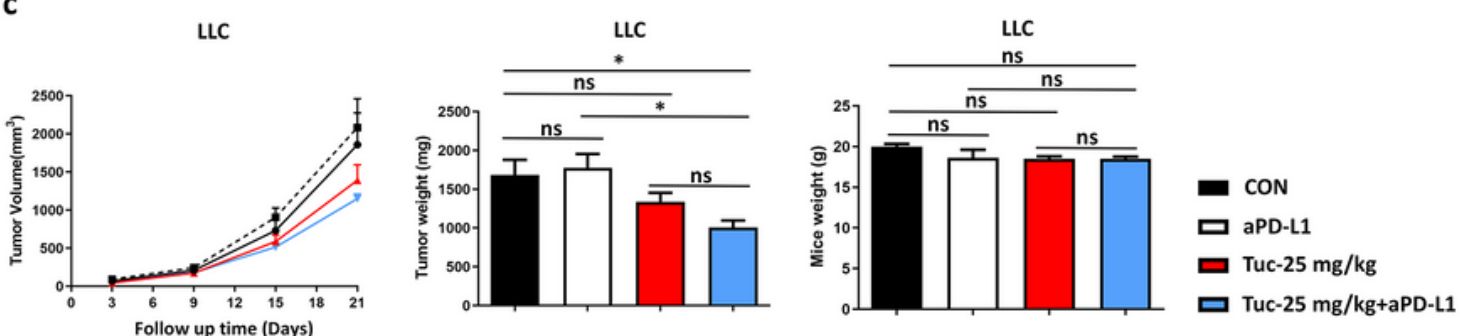

d

CT26
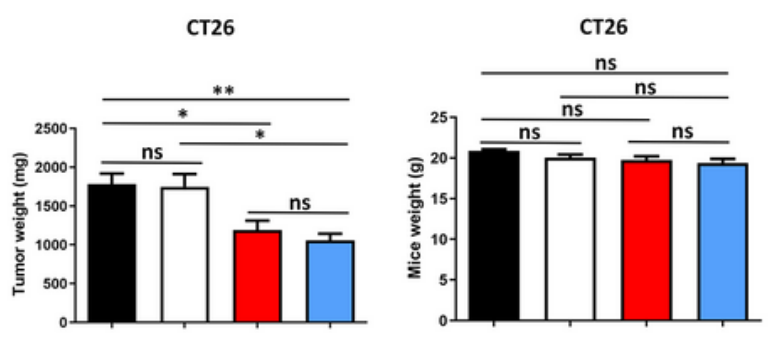

- $\operatorname{CON}$

口 aPD-L1

口 Tuc-25 mg/kg

口 Tuc-25 mg/kg+aPD-L1

e

$4 \mathrm{~T} 1$

uLC

CT26
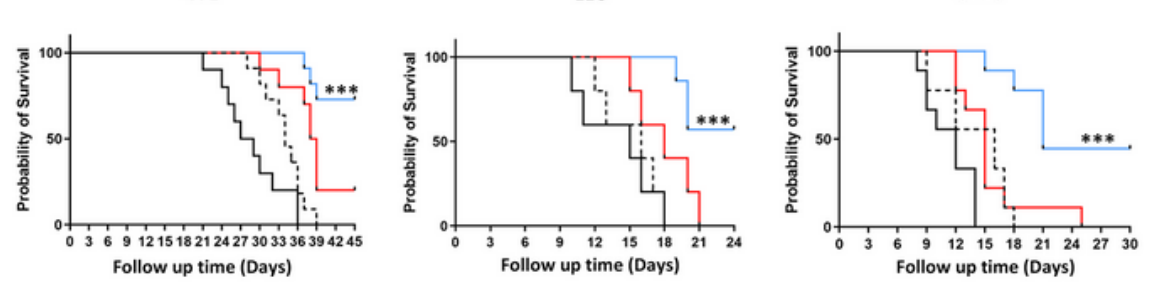

- $\operatorname{CON}$

口 aPD-L1

- Tuc-25 mg/kg

口 Tuc-25 mg/kg+aPD-L1

Follow up time (Days)

\section{Figure 4}

Tucidinostat improves the efficacy of checkpoint blockade and induces a durable response.

(a) Schema of the experiment. Mouse 4T1, LLC, and CT26 cells $\left(5 \times 10^{5}\right.$ cells) were engrafted into the flank of BALB/c or $\mathrm{C} 57 \mathrm{BL} / 6$ mice. When established tumors were palpable 7 days after tumor cells inoculation, mice were treated with vehicle (DMSO, n=7), tucidinostat ( $25 \mathrm{mg} / \mathrm{kg}$, gavage, daily, $\mathrm{n}=7$ ), aPD- 
L1 (200mg, i.p. injection, once every 3 days, $n=7)$, or combination $(n=7)$. Tumors volume were measured with calipers every three days. (b) Tumor growth curves (left) of these mice at day 33 post treatment initiation are shown in 4T1 mouse tumor model. Tumor weight (middle) and mice weight of these mice (right) at day 21 post treatment initiation are shown. (c) Tumor growth curves (left), tumor weight (middle), and mice weight (right) of these mice at day 21 post treatment initiation are shown in LLC mouse tumor model. (d) Tumor growth curves (left), tumor weight (middle), and mice weight (right) of these mice at day 21 post treatment initiation are shown in CT26 mouse tumor model. (e) Kaplan-Meier survival curves of these mice are shown in 4T1, LLC, and CT26 mouse tumor models.

The error bars indicate \pm SEM. ${ }^{*} \mathrm{P}<0.05,{ }^{*} \mathrm{P}<0.01,{ }^{*} * \mathrm{P}<0.001$ by one-way ANOVA or log rank test. ns: not significant. CON: control group; Tuc: tucidinostat; aPD-L1: anti-programmed cell death ligand 1 antibody. 
Figure 5. Tucidinostat and checkpoint blockade increase $\mathrm{CD}^{+} \mathrm{T}$ cells infiltration and $\mathrm{M} 1$ polarization in tumors.

a
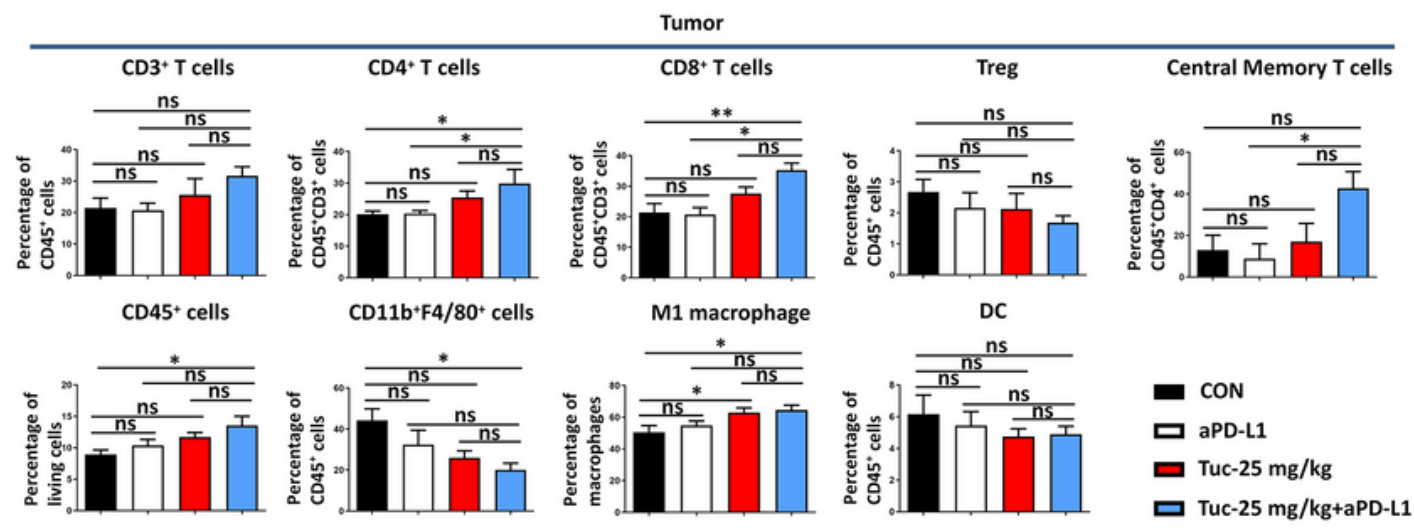

b
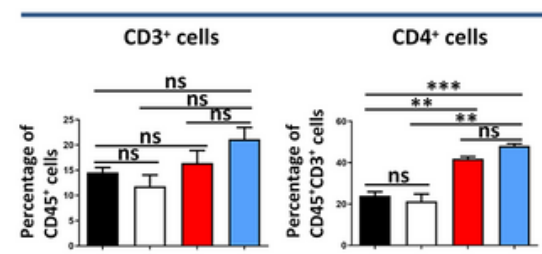

Lymph node
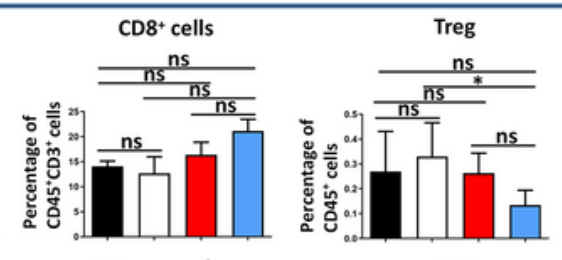

Central Memory T cells

$\mathrm{CD}^{4} 5^{+}$cells

$\mathrm{CD} 11 \mathrm{~b}+\mathrm{F} 4 / 80^{+}$cells

M1 macrophage
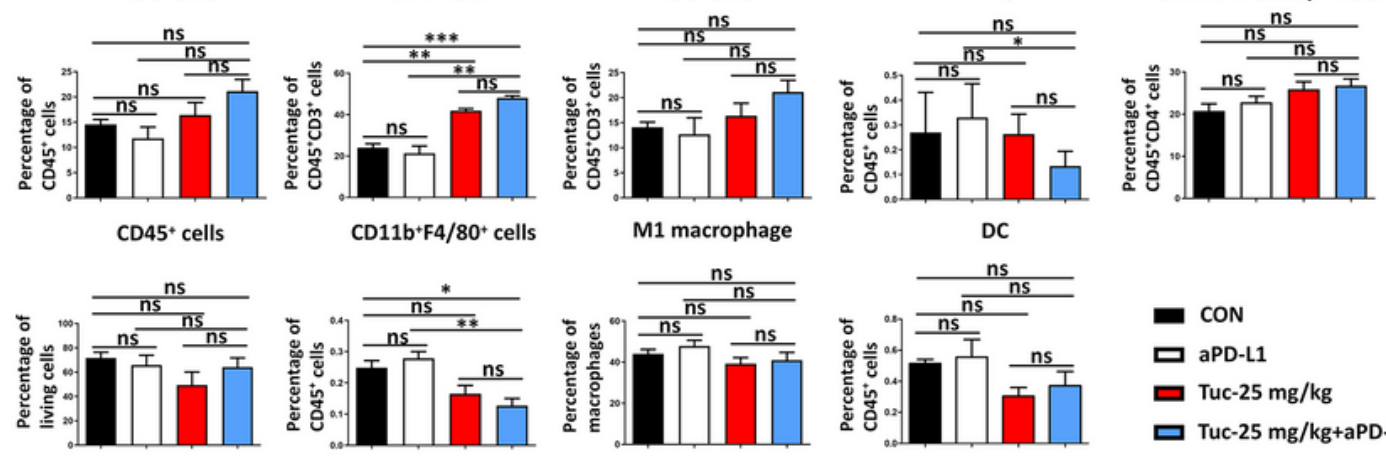

CON
ㅁ aPD-L1
Tuc-25 mg/kg

口 Tuc-25 mg/kg+aPD-L1

\section{Figure 5}

Tucidinostat and checkpoint blockade increase $C D 8^{+} \mathrm{T}$ cells infiltration and M1 polarization in tumors.

Flow cytometric quantification of lymphocytes $\left(\mathrm{CD} 45^{+}\right)$, total T cells $\left(\mathrm{CD} 45^{+} \mathrm{CD} 3^{+}\right), \mathrm{CD} 4^{+} \mathrm{T}$ cells $\left(\mathrm{CD} 45^{+} \mathrm{CD}^{+} \mathrm{CD}^{+}\right), \mathrm{CD}^{+} \mathrm{T}$ cells $\left(\mathrm{CD} 45^{+} \mathrm{CD}^{+} \mathrm{CD} 8^{+}\right)$, Treg cells $\left(\mathrm{CD} 45^{+} \mathrm{CD} 3^{+} \mathrm{CD} 4^{+} \mathrm{CD} 25^{+}\right)$, Central Memory T cells $\left(\mathrm{CD}^{+} \mathrm{CD}^{+} \mathrm{CD} 44^{+} \mathrm{CD} 62 \mathrm{~L}^{+} \mathrm{CD} 197^{+}\right)$, Macrophages $\left(\mathrm{CD} 45^{+} \mathrm{CD} 11 \mathrm{~b}^{+} \mathrm{F} 4 / 80^{+}\right), \mathrm{M} 1$ macrophages 
$\left(\mathrm{CD} 45^{+} \mathrm{CD} 11 \mathrm{~b}^{+} \mathrm{F} 4 / 80^{+} / \mathrm{MHC}^{-} \mathrm{I}^{+}\right)$, and $\mathrm{DC}$ cells $\left(\mathrm{CD} 45^{+} \mathrm{CD} 11 \mathrm{~b}^{-} \mathrm{CD} 11 \mathrm{c}^{+}\right)$in tumor parenchyma (a) and tumor drainage lymph nodes (b) from CT26 tumor-bearing mice on day 21 following different treatment groups (vehicle, tucidinostat, aPD-L1, and combination).

The error bars indicate \pm SEM. ${ }^{*} \mathrm{P}<0.05,{ }^{\star \star} \mathrm{P}<0.01,{ }^{\star \star *} \mathrm{P}<0.001$ by one-way ANOVA. ns: not significant. dLNs: drainage lymph nodes; CON: control group; Tuc: tucidinostat; aPD-L1: anti-programmed cell death ligand 1 antibody.

Figure 6. CCL5 and CD8+ $\mathrm{T}$ cells are required for antitumor immunity induced by tucidinostat and aPD-L1 blockade.

a

Tumor implantation Cell lines: CT26 cell

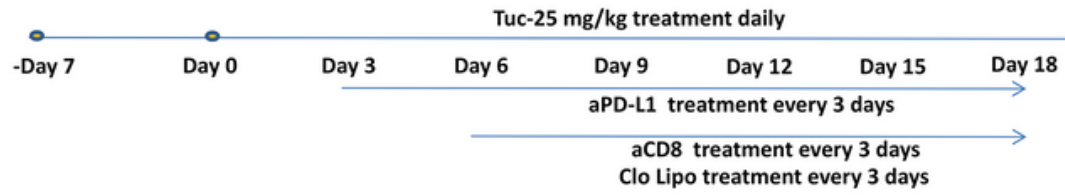

b
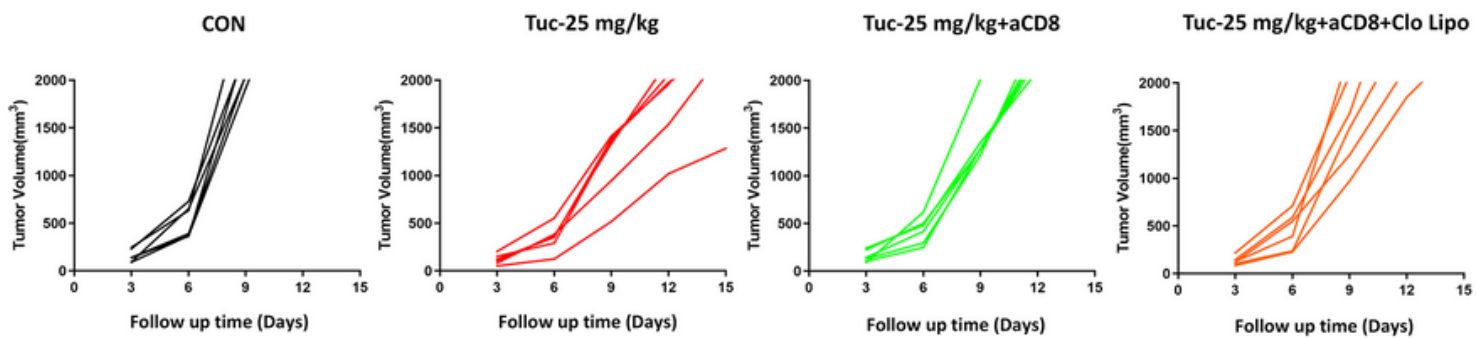

Tuc-25 mg/kg+aPD-L1

Tuc-25 mg/kg +aPD-L1+aCD8

Tuc-25 mg/kg +aPD-L1++Clo Lipo
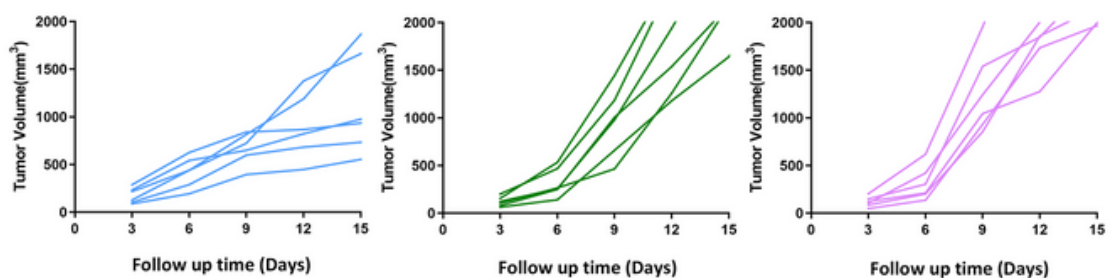

c
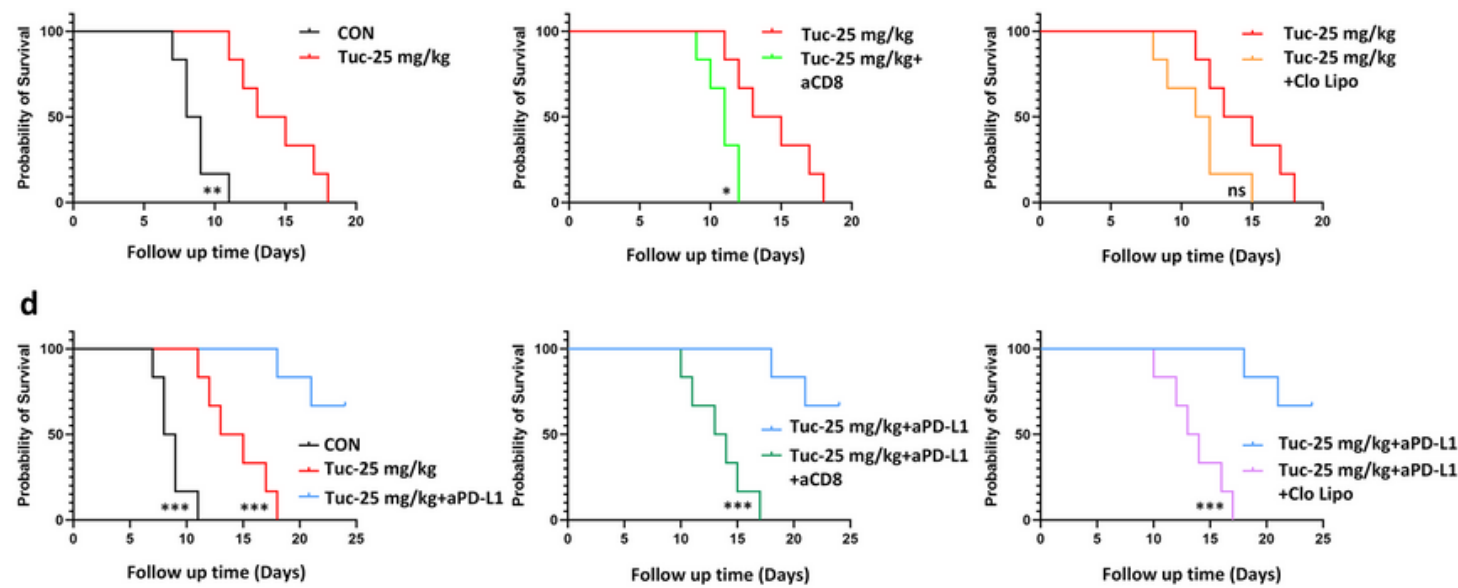


\section{Figure 6}

\section{$\mathrm{CD}^{+} \mathrm{T}$ cells are required for antitumor immunity induced by tucidinostat and aPD-L1 blockade.}

(a) Schema of the experiment. Mouse CT26 cells $\left(5 \times 10^{5}\right.$ cells) were engrafted into the flank of BALB/c mice. When established tumors were palpable 7 days after tumor cells inoculation, mice were treated with tucidinostat ( $25 \mathrm{mg} / \mathrm{kg}$, gavage, daily, $\mathrm{n}=7) \pm$ aPD-L1 (200 $\mu$ g, i.p. injection, once every 3 days, $\mathrm{n}=7$ ) regimen as indicated, in the presence or absence of aCD8 $(200 \mu \mathrm{g}$, i.p. injection, once every 3 days, $n=7)$ or Clodronate liposomes (Macrophage depletion; $1.4 \mathrm{mg} / 20 \mathrm{~g}$ body weight, i.p. injection, once every 3 days, $\mathrm{n}=7$ ). The following groups were included: vehicle; tucidinostat; tucidinostat+aCD8; tucidinostat+Clodronate liposomes; tucidinostat+aPD-L1; tucidinostat+aPD-L1+aCD8; tucidinostat+aPDL1+Clodronate liposomes. Tumors volume were measured with calipers every three days. (b) Tumor growth curves of these mice are shown in CT26 mouse tumor model. (c) Survival differences between the tucidinostat group and the tucidinostat $+\mathrm{aCD} 8 /$ Clodronate liposomes group were evaluated. (d) Survival differences between the tucidinostat+aPD-L1 group and the tucidinostat+aPD-L1+aCD8/Clodronate liposomes group were evaluated.

The error bars indicate \pm SEM. ${ }^{*} \mathrm{P}<0.05,{ }^{*} \mathrm{P}<0.01,{ }^{\star} * \star \mathrm{P}<0.001$ by one-way ANOVA or log rank test. ns: not significant. CON: control group; Tuc: tucidinostat; aPD-L1: anti-programmed cell death ligand 1 antibody; aCD8: anti-CD8 antibody; Clo Lipo: Clodronate liposomes. 
Figure 7. Tucidinostat increases the expression of MHC II and co-stimulatory molecules on human monocytes.

a

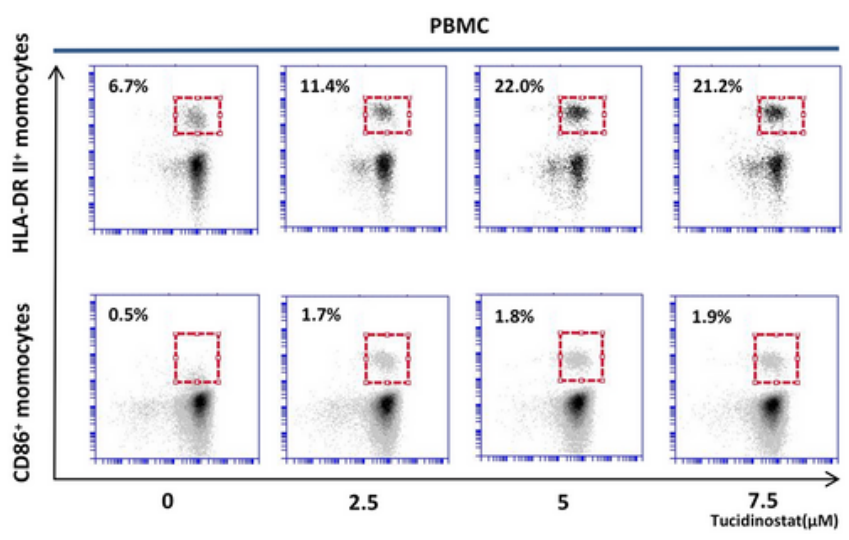

b

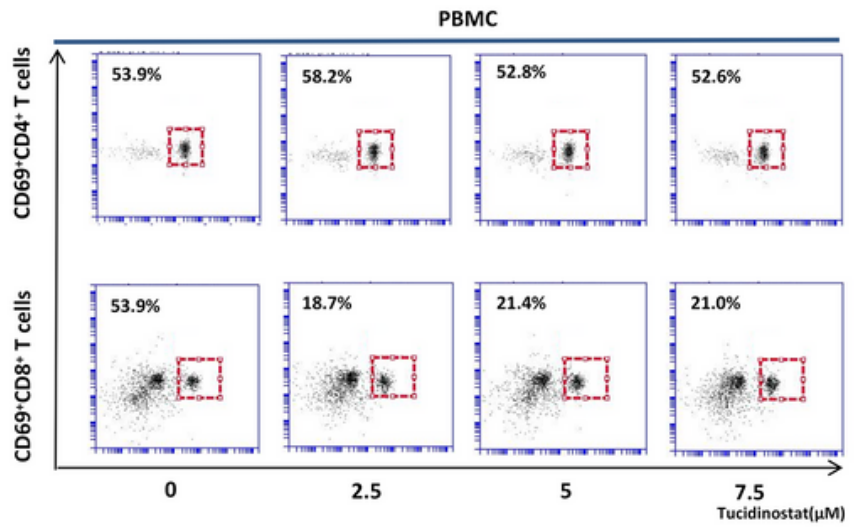

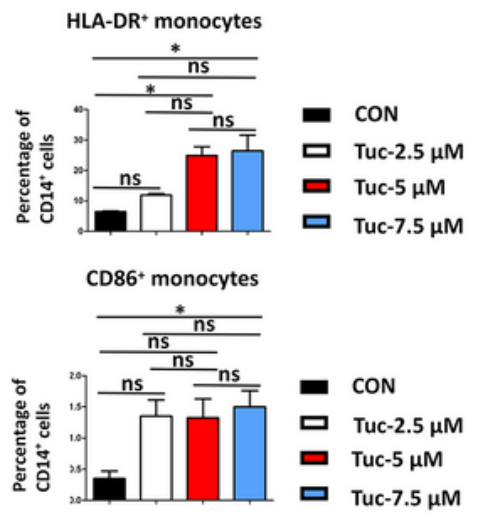

CD69+ CD4+T cells
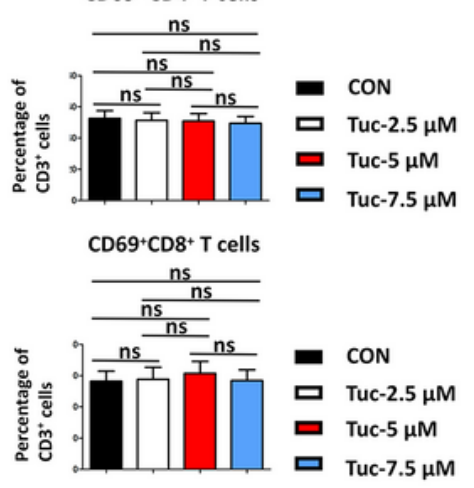

Figure 7

Tucidinostat increases the expression of the co-stimulatory molecules on human monocytes.

(a) PBMCs from NSCLC patients $(n=5)$ were cultured with increasing concentrations of tucidinostat $(2.5 \mu \mathrm{M}, 5 \mu \mathrm{M}, 7.5 \mu \mathrm{M})$ for $24 \mathrm{~h}$, respectively. Whereafter, the phenotype of peripheral blood monocytes $\left(\mathrm{CD} 14^{+} \mathrm{CD} 11 \mathrm{~b}^{+}\right)$were assessed by FACS. Representative cytograms (left) or summary histograms (right) 
for the expression levels of HLA-DR and CD86 on gated monocytes within the PBMCs. (b) PBMCs from NSCLC patients were cultured with increasing concentrations of tucidinostat $(2.5 \mu \mathrm{M}, 5 \mu \mathrm{M}, 7.5 \mu \mathrm{M})$ for $24 \mathrm{~h}$, respectively. Whereafter, the phenotype of $\mathrm{CD} 4^{+} \mathrm{T}$ cells $\left(\mathrm{CD} 3^{+} \mathrm{CD} 4^{+}\right)$and $\mathrm{CD} 8^{+} \mathrm{T}$ cells $\left(\mathrm{CD} 3^{+} \mathrm{CD} 8^{+}\right)$ were assessed by FACS. Representative cytograms (left) or summary histograms (right) for the expression levels of $\mathrm{CD} 69$ on gated $\mathrm{CD} 4^{+}$and $\mathrm{CD} 8^{+} \mathrm{T}$ cells within the PBMCs.

The error bars indicate \pm SEM. ${ }^{*} \mathrm{P}<0.05,{ }^{*} \mathrm{P}<0.01,{ }^{\star} * \star \mathrm{P}<0.001$ by one-way ANOVA. $n s$ : not significant. CON: control group; Tuc: tucidinostat; PBMC: peripheral blood mononuclear cell; NSCLC: non-small-cell lung cancer.

\section{Supplementary Files}

This is a list of supplementary files associated with this preprint. Click to download.

- SupplementaryFigure1.tif

- SupplementaryFigure2.tif

- SupplementaryFigure3.tif

- SupplementaryFigure4.tif

- SupplementaryFigure5.tif 